\title{
Observing Exoplanets with High Dispersion Coronagraphy. I. The Scientific Potential of Current and Next-generation Large Ground and Space Telescopes
}

\author{
Ji Wang ${ }^{1}$, Dimitri Mawet ${ }^{1}$, Garreth Ruane ${ }^{1}$, Renyu $\mathrm{Hu}^{2,3}$, and Björn Benneke ${ }^{3}$ \\ ${ }^{1}$ Department of Astronomy, California Institute of Technology, MC 249-17, 1200 E. California Boulevard, Pasadena, CA 91106, USA; ji.wang@ caltech.edu \\ 2 Jet Propulsion Laboratory, California Institute of Technology, Pasadena, CA 91109, USA \\ ${ }^{3}$ Division of Geological and Planetary Sciences, California Institute of Technology, Pasadena, CA 91125, USA \\ Received 2016 October 24; revised 2017 February 15; accepted 2017 February 16; published 2017 March 30
}

\begin{abstract}
Direct imaging of exoplanets presents a formidable technical challenge owing to the small angular separation and high contrast between exoplanets and their host stars. High Dispersion Coronagraphy (HDC) is a pathway to achieve unprecedented sensitivity to Earth-like planets in the habitable zone. Here, we present a framework to simulate HDC observations and data analyses. The goal of these simulations is to perform a detailed analysis of the trade-off between raw star light suppression and spectral resolution for various instrument configurations, target types, and science cases. We predict the performance of an HDC instrument at Keck observatory for characterizing directly imaged gas-giant planets in near-infrared bands. We also simulate HDC observations of an Earth-like planet using next-generation ground-based (TMT) and spaced-base telescopes (HabEx and LUVOIR). We conclude that ground-based ELTs are more suitable for HDC observations of an Earth-like planet than future space-based missions owing to the considerable difference in collecting area. For ground-based telescopes, HDC observations can detect an Earth-like planet in the habitable zone around an M-dwarf star at $10^{-4}$ star light suppression level. Compared to the $10^{-7}$ planet/star contrast, HDC relaxes the star light suppression requirement by a factor of $10^{3}$. For space-based telescopes, detector noise will be a major limitation at spectral resolutions higher than $10^{4}$. Considering detector noise and speckle chromatic noise, $R=400$ (1600) is the optimal spectral resolutions for HabEx (LUVOIR). The corresponding star light suppression requirement to detect a planet with planet/star contrast $=6.1 \times 10^{-11}$ is relaxed by a factor of 10 (100) for HabEx (LUVOIR).
\end{abstract}

Key words: brown dwarfs - instrumentation: spectrographs - planetary systems techniques: high angular resolution - techniques: spectroscopic

\section{Introduction}

Out of the thousands of exoplanets detected to date, the few that have been directly imaged are excellent targets for studying orbital configurations (Millar-Blanchaer et al. 2015; Maire et al. 2015; Pueyo et al. 2015; Zurlo et al. 2016) and atmospheric chemical compositions (Konopacky et al. 2013; Oppenheimer et al. 2013; Bonnefoy et al. 2016; Rajan et al. 2015). However, direct imaging and characterization faces several technical challenges owing to the small angular separation and high contrast between exoplanets and their host stars. High-contrast imaging (HCI) systems mitigate these effects by suppressing diffracted star light that may otherwise overwhelm the planet signal with an extreme adaptive optics (AOs) system and a coronagraph. Current state-of-the-art HCI instruments, such as the Gemini Planet Imager at the Gemini South telescope (Macintosh et al. 2014) and SPHERE at the Very Large Telescope (Beuzit et al. 2008), are able to achieve a suppression level at a few tenths of an arcsecond of better than $10^{-4}$, which allows for the detection of gas-giant planets and brown dwarfs orbiting nearby young stars (e.g., Macintosh et al. 2015; Wagner et al. 2016).

Star light suppression can be further improved by coupling a high-resolution spectrograph (HRS) with a coronagraphic system (Sparks \& Ford 2002; Riaud \& Schneider 2007; Kawahara \& Hirano 2014; Snellen et al. 2015; Lovis et al. 2017). In this High Dispersion Coronagraphy (HDC) scheme, the coronagraphic component serves as a spatial filter to separate the light from the star and the planet. The HRS serves as spectral filter by taking advantage of differences in spectral features between the stellar spectrum and the planetary spectrum, e.g., different absorption lines and radial velocities (RVs).

That HRS can be used as a way of spectral filtering has been successfully demonstrated by several teams. For example, high-resolution transmission spectroscopy has been used to detect molecular gas in the atmospheres of transiting planets (Snellen et al. 2010; Birkby et al. 2013; de Kok et al. 2013). At a high spectral resolution, resolved molecular lines can be used to study day- to night-side wind velocity (Snellen et al. 2010) and to validate 3D exoplanet atmosphere models (Kempton et al. 2014). For planets detected via $\mathrm{RV}$, the spectral lines that are due to the planet can be separated from stellar lines with their drastically different RVs $\left(\gtrsim 50 \mathrm{~km} \mathrm{~s}^{-1}\right)$. Thus, the RV of the planet itself may be measured to break the degeneracy between the true planet mass and orbital inclination (Brogi et al. 2012, 2013, 2014; Lockwood et al. 2014). Moreover, HRS permits the detailed study of spectral lines arising from a planet's atmosphere. This approach led to the first measurement of a planet's rotational velocity (Snellen et al. 2014). With time-series HRS, surface features such as cloud or spot coverage may be inferred via Doppler imaging, which has been demonstrated on the closest brown dwarf system, Luhman 16 AB (Crossfield et al. 2014).

As showcased by the examples above, HRS may be used to detect planets that are $\sim 10^{-4}$ times as bright as their host stars. When coupled with a state-of-the-art HCI system capable of reaching star light suppression levels of $\sim 10^{-4}$, an HDC instrument is sensitive to much fainter planets. Meanwhile, relatively bright planets may be observed at a 


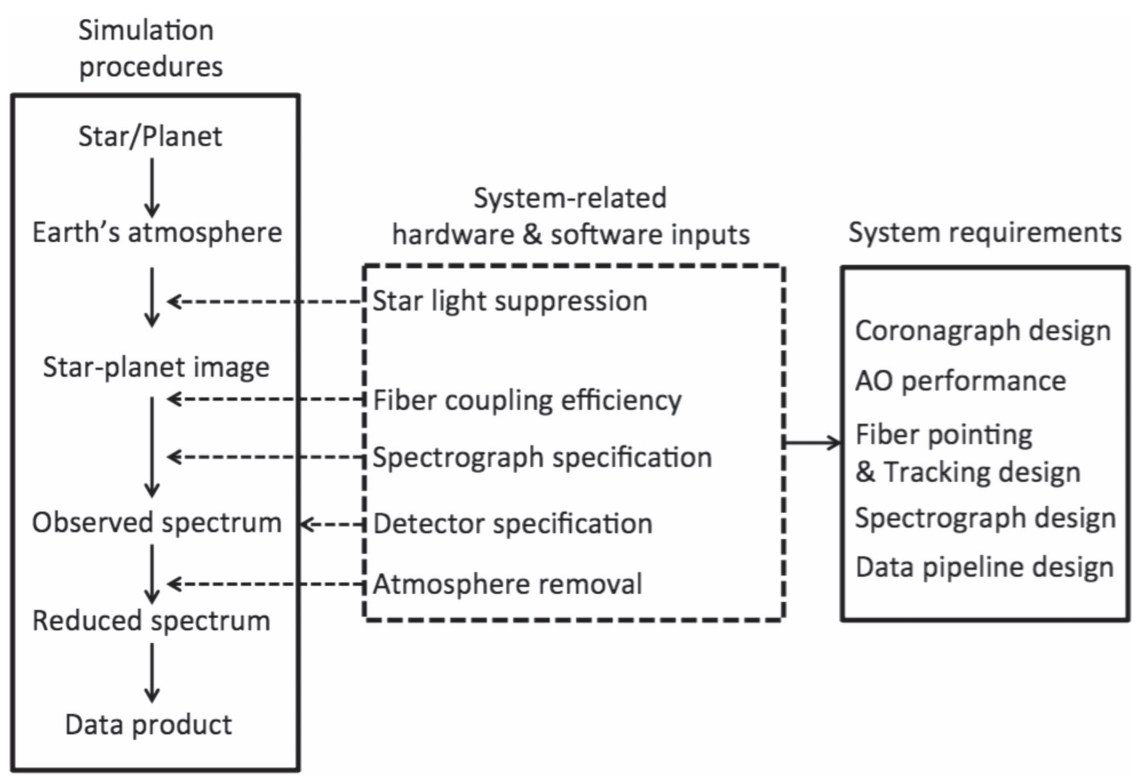

Figure 1. Flow chart of the simulation of an HDC instrument. Photons from star and planet go through an HCI instrument. Residual star light and planet light is picked up by a single-mode fiber, which feeds the light into an HRS instrument. Spectra are simulated with detector noise and then reduced into a data product, e.g., CCF (see Section 2.2). The atmospheric effect is optional depending on ground-based or space-based observation. The simulation pipeline provides a way of setting system requirements for an HDC instrument and understanding the fundamental limit of the HDC technique.

higher signal-to-noise ratio $(\mathrm{S} / \mathrm{N})$ that allows for the physical and chemical processes taking place in their atmospheres to be studied in greater detail. Here, we develop a framework to simulate the performance of an HDC instrument. Although similar calculations have been performed as part of previous studies (Sparks \& Ford 2002; Riaud \& Schneider 2007; Kawahara \& Hirano 2014; Snellen et al. 2015; Lovis et al. 2017), a thorough end-to-end simulation that explores the $\mathrm{S} / \mathrm{N}$ trade space between spectral resolution and star light suppression for ground-based and space-based observations is lacking. In this paper, we simulate a variety of HDC instruments that are either under development or in the conceptual design phase and quantify their potential for detecting new planets as well as particular molecular species in the atmosphere of known planets (e.g., Proxima Cen b, 51 Eri b, and HR 8799 e) and hypothetical Earth-like planets around stars of different spectral types.

The paper is organized as follows. We outline the procedure used to simulate the performance of an HDC instrument for detecting and characterizing exoplanets in Section 2. The planned Keck HDC instrument is briefly described in Section 3. We study the prospects of using the Keck HDC instrument to observe previously imaged exoplanets in Section 4. HDC observations of potential Earth-like planets (e.g., Proxima Cen b) in the habitable zone of $\mathrm{M}$ dwarfs are investigated in Section 5 for current and next-generation extremely large telescopes. Observing Earth-like planets around solar-type stars with future space telescopes is considered in Section 6. A summary and discussion are provided in Section 7.

\section{HDC Fundamental Trade-off Analysis}

\subsection{Simulating the Observations}

In this section, we describe our workflow to simulate the end-to-end performance of an HDC system from the intrinsic spectrum of a planet and star to the measured spectrum and the subsequent post-processing. The goal of these simulations is to perform a detailed analysis of the trade-off between raw star light suppression and spectral resolution for various instrument configurations, target types, and science cases. Figure 1 shows a flow chart to illustrate the procedure and the system-related inputs to the simulation. The resulting data products, e.g., cross-correlation fuction (CCF) and their quality (e.g., $\mathrm{S} / \mathrm{N}$ ), will inform observation strategies and system requirements, including the coronagraph design and the performance of the AOs system.

\subsubsection{Generating Spectra of Stars and Planets}

Gas-giant planet spectra consisting of all molecular species are derived from the published BT-Settl grids $^{4}$ (Baraffe et al. 2015). The grids cover effective temperatures $\left(T_{\text {eff }}\right)$ from 1200 to $7000 \mathrm{~K}$. For $T_{\text {eff }}$ outside of this range, we use the BTSettl grids with Caffau et al. (2010) solar abundances ${ }^{5}$ $\left(400 \mathrm{~K}<T_{\text {eff }}<8000 \mathrm{~K}\right)$. The stellar spectra used in our simulations are also derived from these grids, which cover the $T_{\text {eff }}$ and $\log (g)$ range of host stars considered here. If necessary, the planet and star fluxes are scaled to match the observed absolute flux.

High-resolution spectra discerning the individual contributions of the molecular absorbers $\mathrm{H}_{2} \mathrm{O}, \mathrm{CO}$, and $\mathrm{CH}_{4}$ are simulated using the SCARLET model (Benneke \& Seager 2013; Benneke 2015). In this work, SCARLET first iteratively computes the line-by-line radiative transfer and atmospheric chemistry to converge to a self-consistent vertical temperature structure and molecular composition. To isolate the contribution from individual molecules, we then artificially remove all opacities in the atmosphere except for the opacity of the respective molecular absorber and the collision-induced absorption in the simulation of the planets' thermal emission spectra. In this way, we compute emission spectra for each of the molecular absorbers individually. The SCARLET model

\footnotetext{
4 https://phoenix.ens-lyon.fr/Grids/BT-Settl/CIFIST2011_2015/FITS/

5 https://phoenix.ens-lyon.fr/Grids/BT-Settl/CIFIST2011/SPECTRA/
} 
considers the molecular opacities of $\mathrm{H}_{2} \mathrm{O}, \mathrm{CH}_{4}, \mathrm{NH}_{3}, \mathrm{HCN}$, $\mathrm{CO}, \mathrm{CO}_{2}$ and $\mathrm{TiO}$ from the high-temperature ExoMol database (Tennyson \& Yurchenko 2012), and $\mathrm{O}_{2}, \mathrm{O}_{3}, \mathrm{OH}, \mathrm{C}_{2} \mathrm{H}_{2}, \mathrm{C}_{2} \mathrm{H}_{4}$, $\mathrm{C}_{2} \mathrm{H}_{6}, \mathrm{H}_{2} \mathrm{O}_{2}$, and $\mathrm{HO}_{2}$ from the HITRAN database (Rothman $\&$ Gordon 2009). Absorption by the alkali metals (Li, Na, K, $\mathrm{Rb}$, and $\mathrm{Cs}$ ) is modeled based on the line strengths provided in the VALD database (Piskunov et al. 1995) and in the $\mathrm{H}_{2}$ broadening prescription provided in Burrows \& Volobuyev (2003). Collision-induced broadening from $\mathrm{H}_{2} / \mathrm{H}_{2}$ and $\mathrm{H}_{2} / \mathrm{He}$ collisions is computed following Borysow (2002).

The spectra of Earth-like exoplanets, on the other hand, are generated by an atmospheric chemistry and radiative transfer model (Hu et al. 2012a, 2012b, 2013; Hu \& Seager 2014). We first calculate the molecular abundance as a function of altitude, controlled by photochemical and disequilibrium chemistry processes. The details of the model are described in $\mathrm{Hu}$ et al. (2012a) and the molecular abundances have been validated against measurements on Earth.

We include the effects of clouds in the resulting spectra by averaging two scenarios: a cloud-free scenario where we assume a clear atmosphere, and a high-cloud scenario where we assume a reflective $\mathrm{H}_{2} \mathrm{O}$ cloud at $9-13 \mathrm{~km}$. This procedure produces a continuum albedo of $\sim 0.3$ and provides a realistic estimate of the strength of spectral features, similar to Des Marais et al. (2002). Eighth-order Gaussian integration is used to calculate the contribution of the whole planetary disk for both the reflected light and thermal emission. We include the opacities of $\mathrm{CO}_{2}, \mathrm{O}_{2}$, $\mathrm{H}_{2} \mathrm{O}, \mathrm{CH}_{4}, \mathrm{O}_{3}$, and $\mathrm{N}_{2} \mathrm{O}$ and calculate the planetary flux at a spectral resolution of $R=\lambda / \Delta \lambda=500,000$, which is high enough to resolve individual spectral lines of the aforementioned species over $\lambda=0.5-5 \mu \mathrm{m}$. The resulting spectra are then expressed as albedo and scaled with the planet's size within the reasonable range for terrestrial planets.

\subsubsection{Spectrum of Earth's Atmosphere}

Telluric and sky emission lines are included in the simulation to account for additional photon loss, near-infrared background noise, and potential confusion between molecules that appear in both the planet's and Earth's atmosphere, e.g., $\mathrm{H}_{2} \mathrm{O}$ and $\mathrm{O}_{2}$. We use the Maunakea sky transmission ${ }^{6}$ and emission spectra, ${ }^{7}$ available from the Gemini observatory website (Lord 1992), with wavelength coverage of $0.9-5.6 \mu \mathrm{m}$. A water column density of $1.6 \mathrm{~mm}$ and airmass of 1.5 is assumed. Since we also consider telluric absorption at shorter wavelengths, we also use telluric absorption data from the National Solar Observatory for wavelengths shorter $\operatorname{than}^{8} 0.9 \mu \mathrm{m}$.

\subsubsection{Simulation Procedure}

An HDC instrument contains two main components, a coronagraph and an HRS, which are linked by a set of singlemode fibers: a planet fiber, a star fiber, and/or a sky fiber. One end of these fibers is located at the image plane after the coronagraph, and the other end of the fibers is at the entrance slit of the spectrograph. The star fiber and sky fiber provide calibration spectra in data reduction that are described in Section 2.2. Following Figure 1, light from the star and planet

\footnotetext{
6 http://www.gemini.edu/sciops/telescopes-and-sites/observing-conditionconstraints /ir-transmission-spectra

7 http://www.gemini.edu/sciops/telescopes-and-sites/observing-conditionconstraints/ir-background-spectra

8 diglib.nso.edu/ftp.html
}

go through a coronagraph and form an image. The fiber at the planet location leads the planet light, as well as residual star light, into the spectrograph. The detector records the planet spectrum along with the contaminating stellar spectrum. We note that atmospheric effect, i.e., absorption and emission, is only considered for ground-based observations,

$$
f_{\text {detector }}=\left(f_{\text {planet }}+f_{\text {star }} \times C\right) \times f_{\text {transmission }}+f_{\text {sky }} .
$$

We simulate the signal recorded on a detector as described in Equation (1). Flux from a star and a planet is in the unit of $\mathrm{W} \mathrm{m}^{-2} \mu \mathrm{m}^{-1}$ at a reference height $\left(d_{\text {ref }}\right)$. We calculate the incident star and planet photon flux on the detector, $f_{\text {star }}$ and $f_{\text {planet }}$, with the following equation: $f=F \times\left(d_{\text {ref }} / d\right)^{2} \times$ $A \times \Delta \lambda \times \eta \times t_{\exp } / h \nu$, where $F$ is the flux at the reference height $\left(d_{\text {ref }}\right), d$ is distance between the star-planet system and an observer, $\mathrm{A}$ is telescope receiving area, $\Delta \lambda$ is the wavelength coverage per wavelength bin, $\eta$ is the telescope and instrument end-to-end throughput, $t_{\exp }$ is the exposure time, $h$ is the Planck constant, and $\nu$ is the frequency of a photon.

At the image plane after a coronagraph, stellar flux is suppressed by a factor $C$, a parameter we denote as star light suppression factor, i.e., the fraction of the total star light that couples into the fiber.

Both stellar and planetary spectra are rotationally broadened. The effect of rotation broadening is calculated by summing spectra from surface grids that are evenly spaced in longitudinal and latitudinal direction. The rotationally broadened spectra are then multiplied by the Earth's atmosphere transmission spectrum ( $\left.f_{\text {transmission }}\right)$ for ground-based observation. The Earth's atmosphere transmission spectrum is unitless and normalized to unity, with zero meaning entirely opaque and one meaning entirely transmissive. For space-based observations, $f_{\text {transmission }}$ is set to unity.

The spectra are then broadened by the instrumental line spread function. The instrumental broadening is approximated by convolving a spectrum with a normalized Gaussian core with a full width at half maximum of one spectral resolution element, which is $\lambda_{0} / R$, where $\lambda_{0}$ is the central wavelength and $R$ is the spectral resolution of a spectrograph.

The broadened spectra are then added by sky emission spectrum $\left(F_{\text {emission }}\right)$, which is also broadened at a given spectral resolution. The sky emission is in the unit of photons $\mathrm{s}^{-1} \operatorname{arcsec}^{-2} \mathrm{~m}^{-2} \mu \mathrm{m}^{-1}$. We calculate the incident photon flux from the sky emission on the detector using the following equation: $f_{\text {emission }}=F_{\text {emission }} \times t_{\exp } \times \theta^{2} \times A \times \Delta \lambda$, where $\theta^{2}$ is the projected area of sky to the input fiber fundamental mode size, which we assume to be $\left(1.0 \lambda_{0} / D\right)^{2}$, where $\lambda_{0}$ is the central wavelength and $D$ is the telescope aperture diameter. For space-based observation, $f_{\text {emission }}$ is set to zero. The simulated spectra are then resampled at the pixel sampling rate per resolution element.

In addition to the spectrum described by Equation (1), we simulate more spectra for subsequent data reduction. For ground-based observation, we simulate sky emission and stellar spectra, assuming there are two dedicated fibers for sky and star. The stellar spectrum can be used to remove atmospheric transmission and/or contaminated stellar lines in the planet spectrum. For example, in the case of ground-based observations of the HR 8799 system, the host star itself is a fastrotating early-type star and thus can be used as a telluric standard to remove atmosphere transmission. In the case of 
ground-based observations of Proxima Cen b, the observed spectrum is a reflection spectrum containing both the star and planet absorption lines and is contaminated by the Earth's atmosphere lines, so that it is necessary to have a separate simultaneous observation of the host star to remove atmospheric transmission and/or contaminated stellar lines in the planet spectrum. For space-based observations, we simulate only the stellar spectrum since the background is negligible.

\subsubsection{Noise Sources}

We include realistic estimates of photon noise, detector readout noise $(\mathrm{RN})$, and dark current based on the performance of a Teledyne $\mathrm{HgCdTe} \mathrm{H} 2 \mathrm{RG}$ infrared detector and a $\mathrm{e} 2 \mathrm{v}$ optical charge-coupled device (CCD). $\mathrm{RN}$ and dark current for the H2RG detector are $2.0 e^{-}$(Fowler-32 readout, personal correspondence with Roger Smith) and $0.002 e^{-} \mathrm{s}^{-1}$ (Blank et al. 2012), respectively. An e2v optical $\mathrm{CCD}^{9}$ has an $\mathrm{RN}$ of $2.0 e^{-}$and a dark current of $0.02 e^{-} \mathrm{hr}^{-1}$,

$$
\delta=\sqrt{f+n_{\exp } \times \mathrm{RN}^{2}+\operatorname{dark} \times t_{\mathrm{exp}}} .
$$

The total noise is calculated by Equation (2), where $\delta$ is the combined noise, $f$ is the photon noise followed by terms for RN and dark current (dark), and $n_{\exp }$ is the number of readout within a total observation time $t_{\text {exp }}$. The number of readouts $n_{\text {exp }}$ is determined by the linear range or detector persistence limit, i.e., the signal level where a new frame needs to be taken in order to avoid nonlinearity or persistence. The exposure time per frame or the number of readouts is usually set by the raw level of star light suppression (instrumental contrast) or sky background emission. We make a conservative assumption that the persistence limit is at 12,000 electrons. During operations such as sky emission removal and telluric or stellar line removal, noises are added in quadrature.

\subsection{Spectral Analyses}

Once the detected spectra are obtained, we perform the data processing steps required to extract the planet signal using the cross-correlation technique (Konopacky et al. 2013; Schwarz et al. 2016). First, the sky emission spectrum is subtracted from the planet spectrum and the planet spectrum is corrected for telluric absorption and stellar lines, which results in a so-called reduced spectrum. We note that since telluric removal is divisive and stellar removal is subtractive, stellar removal needs additional care in the presence of significant planet light and abundant stellar lines (Schwarz et al. 2016). Then, the detected planet spectrum is cross-correlated with a synthetic planet template spectrum. For ground-based observations, the spectra used in the cross-correlation are high-pass filtered to remove the spectral continuum component $(<100$ cycles per micron). For space-based observations of planets whose spectra are dominated by reflected light, we remove the continuum by dividing the reflected light spectrum by the stellar spectrum. The cross-correlation between the reduced spectrum and the synthetic spectrum results in a CCF. The peak of the CCF is compared with the fluctuation level of the CCF (illustrated in Figure 2). We define the CCF S/N as the ratio of the CCF peak value and the rms of the CCF fluctuation. To calculate the rms, we use either the first or the fourth quarter of CCF, whichever is farther awary from the CCF peak. In order to be qualified as a

\footnotetext{
http://www.e2v.com/resources/account/download-datasheet/1364
}

significant detection, we require that (1) the CCF S/N is higher than 3 and (2) the RV of CCF peak is consistent with the input planet RV within one resolution element. Any significant detection of the CCF peak is equivalent to detecting the planet. To detect a certain molecular species in the planet spectrum (e.g., $\mathrm{CO}$ or $\mathrm{H}_{2} \mathrm{O}$ ), we repeat the same process using a synthetic planet template spectrum consisting of only lines from that single molecular species.

For ground-based observations, the detection of the CCF peak is hampered by the Earth's atmosphere. This is especially the case if the molecular species of interest is also present in the Earth's atmosphere, e.g., $\mathrm{O}_{2}, \mathrm{H}_{2} \mathrm{O}$, and $\mathrm{CO}_{2}$. In such cases, the CCF peak could be caused by residuals from the removal of telluric absorption lines. To distinguish the origin of the $\mathrm{CCF}$ peak, we use the fact that the RV of an exoplanet changes by tens of $\mathrm{km} \mathrm{s}^{-1}$ due to its orbital motion and the Earth's barycentric motion, whereas the RV variation of telluric lines stays within tens of $\mathrm{m} \mathrm{s}^{-1}$. To measure an RV change of tens of $\mathrm{km} \mathrm{s}^{-1}$, the spectral resolution needs to be at least a few thousand at moderate $\mathrm{S} / \mathrm{N}$. Therefore we consider only spectral resolutions higher than $R=1000$ for ground-based observations. The ability of a spectrograph to distinguish the signal from an exoplanet and the signal from the Earth's atmosphere in RV space improves with increased spectral resolution. For space-based observations, the spectral resolution may be as low as $R=25$.

\section{Fiber Injection Unit (FIU), Upgraded NIRSPEC, and the Keck Planet Imager and Characterizer (KPIC) at Keck}

While the framework described in Section 2 is a generalpurpose pipeline to simulate the performance of any HDC instruments, we will use the pipeline to study the prospect of the KPIC (Mawet et al. 2016), an HDC instrument that is being developed at Keck telescope.

The KPIC is a four-pronged upgrade of the Keck AOs facility. The first upgrade component is the addition of a highperformance small inner working angle (IWA) $L$-band vortex coronagraph to NIRC2 (Absil et al. 2016). This operation was successfully carried out in 2015 and is now available to the Keck community in shared-risk mode. The upgrade not only came with a brand-new coronagraph focal plane mask, but also a suite of software packages to automate the coronagraph acquisition procedure, including automatic ultraprecise centering (Huby et al. 2015), speckle nulling wavefront control (Bottom et al. 2016), and an open-source python-based data reduction package (Gomez Gonzalez et al. 2016). The second upgrade component is an infrared pyramid wavefront sensor demonstration, and a potential facility for the Keck II AOs system. The third upgrade component is a higher-order deformable mirror paired with the infrared pyramid sensor, followed by a new single-stage coronagraph. Finally, the fourth component of the KPIC is the FIU.

The FIU is at the core of the KPIC instrument upgrade that links the Keck II telescope AO bench to NIRSPEC, the current $R \sim 25,000$ workhorse infrared spectrograph at Keck. The FIU focuses the light from a target of interest into single-mode fibers after the AO system with minimal losses, and the fiber outputs are reformatted to fit the slit plane of NIRSPEC.

In 2018, the UCLA IR laboratory will equip NIRSPEC with a new $5 \mu$ m cutoff, $2048 \times 2048$ pixel $\mathrm{HgCdTe} \mathrm{H} 2 \mathrm{RG}$ detector from Teledyne (Martin et al. 2014). This new device offers reduced read noise and dark current, as well as improved 

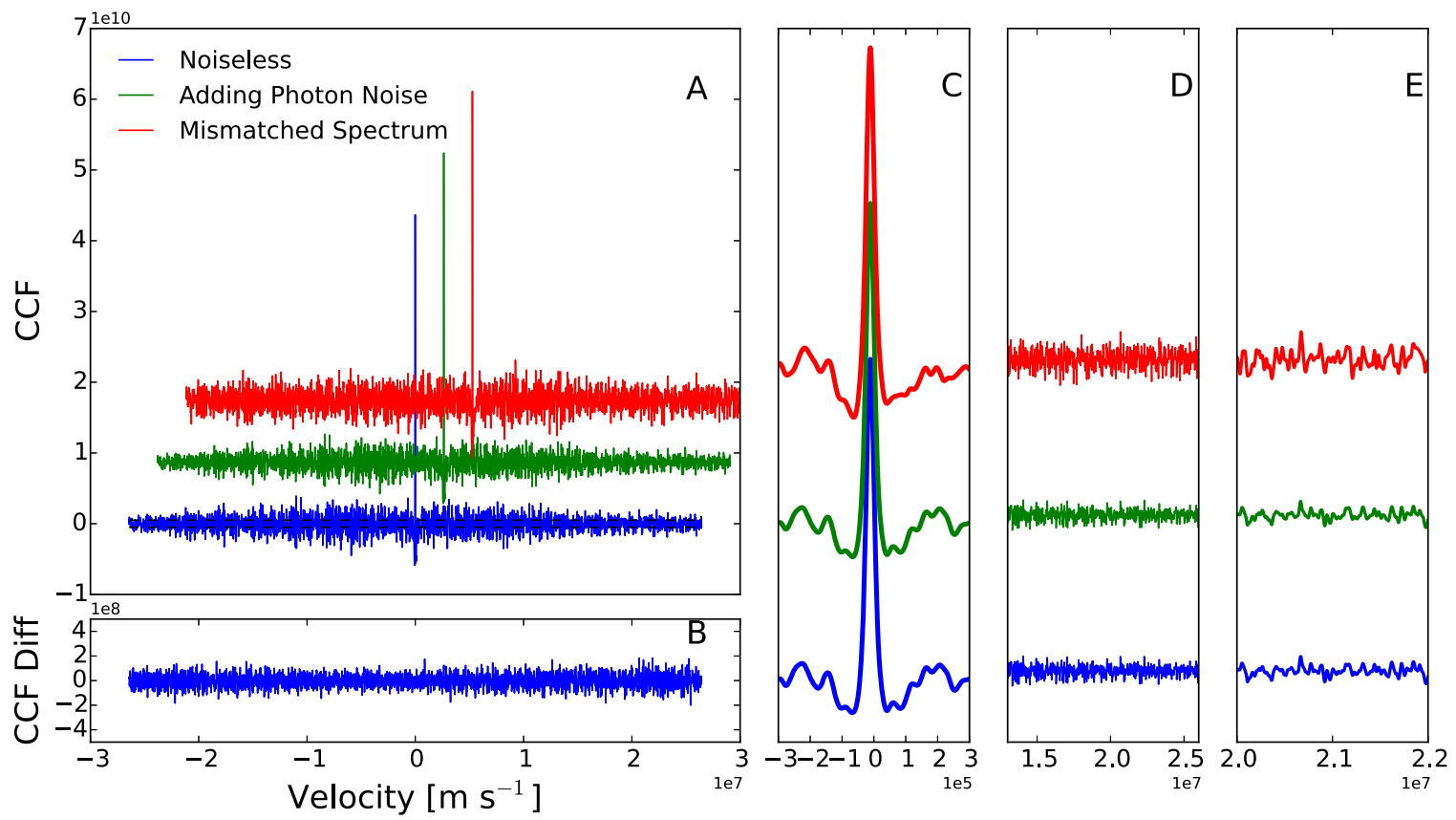

Figure 2. (A) Examples of the cross-correlation function (see Section 4.1 for the definition of different cases). The CCFs are vertically and horizontally offset for visual clarity. The peaks of the CCFs are scaled to the same height to emphasize the different fluctuation level outside the CCF peaks. Dashed lines indicate lower and upper boundaries for the $y$-axis in panel (B). (B) CCF fluctuation due to photon noise, i.e., the difference between the blue and the green CCF in panel (A). When the photon noise is small, the CCF fluctuation due to photon noise is smaller than the CCF fluctuation that is due to intrinsic CCF structures (blue CCF in panel (A)). (C) Close-up for CCF peaks. (D) Close-up for CCF regions where we define the fluctuation level of a CCF. We use the rms of either the first or the fourth quarter to calculate the CCF fluctuation level. (E) Close-up to show CCFs of different cases.

cosmetics, superior flat-fielding, a modest improvement in quantum efficiency in $H$ and especially $K$ band, and the enhanced stability of modern electronics. Most critical for HRS is the H2RGs smaller pixel scale of $18 \mu \mathrm{m}$ (versus $27 \mu \mathrm{m}$ for the existing Aladdin device), which directly improves the spectral resolution with the same grating arrangement from 25,000 to 37,500 with a 0 "' 29 slit and 3-pixel sampling.

The simulations in Section 4 are based on the expected performance of KPIC at various stages of development.

\section{Ground-based Observations of Directly Imaged Planets with HDC}

\section{1. $H R 8799 e$}

Planet e is the most challenging planet to observe of the 4 known planets in the HR 8799 system because of its proximity to the host star $(\simeq 0$ "' 4$)$. Following the methods detailed in Section 2, we simulate observations with an HDC instrument (the FIU and upgraded NIRSPEC) at Keck. The input parameters for the planet, star, telescope, and instrument are provided in Tables 1 and 2.

BT-Settl model spectra are used as the input spectra. We use $T_{\text {eff }}=1200$ and $7400 \mathrm{~K}$ and $\log (g)=3.5$ and 4.5 for the planet and the star, respectively. The metallicity $[\mathrm{Fe} / \mathrm{H}]$ is set to zero for both planet and star.

The flux from the planet and star is adjusted such that the model flux is consistent with the absolute flux measured from photometry. We ensure that the adjusted flux matches the result from Bonnefoy et al. (2016) within the uncertainties (see Figure 3).

We consider two cases: (1) we have perfect knowledge of the planet spectrum, and (2) we have limited information about the intrinsic planet spectrum. In the first case, we use the BT-Settl model spectrum that is used to generate observations
Table 1

Telescope and Instrument Parameters for Simulated Observations of HR 8799 e and 51 Eri b

\begin{tabular}{llc}
\hline \hline Parameter & Value & Unit \\
\hline Telescope aperture & 10.0 & $\mathrm{~m}$ \\
Spectral resolution & 37500 & $\ldots$ \\
$J$-band spectral range & $1.143-1.375$ & $\mu \mathrm{m}$ \\
$H$-band spectral range & $1.413-1.808$ & $\mu \mathrm{m}$ \\
$K$-band spectral range & $1.996-2.382$ & $\mu \mathrm{m}$ \\
$L^{\prime}$-band spectral range & $3.420-4.120$ & $\mu \mathrm{m}$ \\
Exposure time $^{\text {Fiber angular diameter }}$ & 3600 & $\mathrm{~second}$ \\
Wavefront correction residual $^{\mathrm{a}}$ & 1.0 & $\lambda / D$ \\
Telescope+instrument throughput $^{\mathrm{b}}$ & 260 & $\mathrm{~nm}$ \\
Readout noise $^{\text {Dark current }}$ & $10 \%$ & $\ldots$ \\
\hline
\end{tabular}

Notes.

${ }^{a}$ Private communication with Peter Wizinowich.

b This throughput is for $K$ band. Throughputs for other bands are scaled with the Strehl ratio.

as the template. As a result, the input spectrum and the template spectrum are the same. In the second case, a combined molecule-by-molecule spectrum of $\mathrm{CO}, \mathrm{CH}_{4}$, and $\mathrm{H}_{2} \mathrm{O}$ is used as the template. As a result, the input spectrum and the template spectrum are independently generated and may not necessarily be the same.

\subsubsection{Limiting Factors for the CCF $S / N$}

We simulate 100 observations at each star light suppression level and for each band. The median value of these simulations is reported in the following discussion. We consider three 
Table 2

HR 8799 and Planet e

\begin{tabular}{|c|c|c|c|}
\hline Parameter & Value & Unit & References \\
\hline \multicolumn{4}{|l|}{ Star } \\
\hline $\begin{array}{l}\text { Effective temper- } \\
\quad \text { ature }\left(T_{\text {eff }}\right)\end{array}$ & 7193 & $\mathrm{~K}$ & Baines et al. (2012) \\
\hline Surface gravity $(\log g)$ & 4.03 & cgs & Baines et al. (2012) \\
\hline Distance & 39.40 & $\mathrm{pc}$ & van Leeuwen (2007) \\
\hline$V \sin i$ & 37.5 & $\mathrm{~km} \mathrm{~s}^{-1}$ & $\begin{array}{l}\text { Kaye \& Strassmeier } \\
\quad(1998)\end{array}$ \\
\hline Inclination $(i)^{\mathrm{a}}$ & $>\sim 40$ & degree & Wright et al. (2011) \\
\hline Radial velocity & -11.5 & $\mathrm{~km} \mathrm{~s}^{-1}$ & Gontcharov (2006) \\
\hline \multicolumn{4}{|l|}{ Planet } \\
\hline $\begin{array}{l}\text { Effective temper- } \\
\quad \text { ature }\left(T_{\text {eff }}\right)\end{array}$ & $1100-1650$ & $\mathrm{~K}$ & Bonnefoy et al. (2016) \\
\hline Surface gravity $(\log g)$ & $3.5-4.1$ & cgs & Bonnefoy et al. (2016) \\
\hline Metallicity $([M / H])$ & $0.0-0.5$ & $\operatorname{dex}$ & Bonnefoy et al. (2016) \\
\hline$V \sin i^{\mathrm{b}}$ & $<40.0$ & $\mathrm{~km} \mathrm{~s}^{-1}$ & $\begin{array}{l}\text { Konopacky } \\
\text { et al. (2013) }\end{array}$ \\
\hline Inclination $(i)$ & 28 & degree & Soummer et al. (2011) \\
\hline Semimajor axis $(a)$ & $14.94-20.44$ & $\mathrm{au}$ & Zurlo et al. (2016) \\
\hline Radial velocity $^{c}$ & -11.5 & $\mathrm{~km} \mathrm{~s}^{-1}$ & Gontcharov (2006) \\
\hline Angular separation & $0.38-0.52$ & $\operatorname{arcsec}$ & Zurlo et al. (2016) \\
\hline Angular separation in $J$ & $14.6-20.2$ & $\lambda / D$ & Zurlo et al. (2016) \\
\hline Angular separation in $H$ & $11.4-15.7$ & $\lambda / D$ & Zurlo et al. (2016) \\
\hline $\begin{array}{l}\text { Angular separation } \\
\text { in } K_{S}\end{array}$ & $8.4-11.5$ & $\lambda / D$ & Zurlo et al. (2016) \\
\hline $\begin{array}{l}\text { Angular separation } \\
\quad \text { in } L^{\prime}\end{array}$ & $4.7-6.9$ & $\lambda / D$ & Zurlo et al. (2016) \\
\hline Planet/star contrast in $J$ & $2.0 \times 10^{-6}$ & $\cdots$ & $\cdots$ \\
\hline $\begin{array}{l}\text { Planet/star contrast } \\
\text { in } H\end{array}$ & $1.0 \times 10^{-5}$ & $\cdots$ & $\cdots$ \\
\hline $\begin{array}{l}\text { Planet/star contrast } \\
\text { in } K_{S}\end{array}$ & $3.8 \times 10^{-5}$ & $\cdots$ & $\cdots$ \\
\hline $\begin{array}{l}\text { Planet/star contrast } \\
\quad \text { in } L^{\prime}\end{array}$ & $2.1 \times 10^{-4}$ & $\cdots$ & $\cdots$ \\
\hline
\end{tabular}

Notes.

${ }^{\mathrm{a}}$ We adopt $40^{\circ}$ in simulations.

${ }^{\mathrm{b}} \mathrm{We}$ assume a rotational velocity of $15 \mathrm{~km} \mathrm{~s}^{-1}$.

c Assumed to be the same as HR 8799.

scenarios in the CCF S/N calculation (Figure 4). In the $\mathrm{CCF}$ structure-limited case, the $\mathrm{CCF} \mathrm{S} / \mathrm{N}$ is limited by the intrinsic structure in regions where we calculate the noise level. We use the rms of the first quarter or the forth quarter to calculate the noise level of the CCF (see Figure 2 for illustration). If the CCF peak is in the first half of the $\mathrm{CCF}$, then we use the forth quarter for the rms calculation. Otherwise, we use the first quarter for the rms calculation. The velocity span of the CCF is half of the bandwidth times the speed of light, which is the result of the Fourier transform that is used in the CCF calculation.

In theory, the intrinsic $\mathrm{CCF}$ structure can be removed by subtracting the noiseless CCF from the noisy CCF. The remaining noise level is due to photon noise (see Panel (B) in Figure 2), which is the photon-noise-limited case. The limiting photon noise can originate from various sources. At a low level of star light suppression, the dominating noise source is always the photon noise from the star. At deeper star light suppression, the limiting photon noise can be sky background emission (e.g., $L^{\prime}$ band) or the planet itself (e.g., $J, H, K_{S}$ band). The photon-noise-limited case is the most optimistic case in which we have perfect knowledge of the planet and the star.
In practice, however, we do not know the noiseless planet and star spectra a priori, and acoordingly, we do not know the noiseless CCF. Therefore, CCF S/N is almost certainly limited by systematics. In addition to the CCF structure-limited case, we also consider one case in which systematics dominates the $\mathrm{CCF} S / \mathrm{N}$. In the mismatched-spectrum case, we consider a mismatch between the observed and the template planet spectrum. For the observed planet spectrum, we use a BT-Settl spectrum with $T_{\text {eff }}=1200 \mathrm{~K}$ and $\log (g)=3.5$. For the template planet spectrum, we use the combined spectrum of $\mathrm{CO}, \mathrm{CH}_{4}$, and $\mathrm{H}_{2} \mathrm{O}$ as shown in Figure 3. This scenario yields the lowest $\mathrm{CCF} \mathrm{S} / \mathrm{N}$ because of the spectrum mismatch.

Although this case can potentially result in a low $C C F S / N$, it represents an opportunity for atmosphere retrieval: a more probable molecular abundance ratio, $P-T$ profiles may be determined by varying the model parameters to maximize the CCF peak. It highlights the importance of planet spectrum modeling and a good understanding of the systematics associated with the cross-correlation method.

The three limiting cases represent the different stages of spectral retrieval. From a reduced spectrum, a template (most likely mismatched) is used in the cross-correlation, which results in a $\mathrm{CCF}$ peak, assuming the template resembles the planet spectrum in the reduced spectrum. The result of this stage is equivalent to the mismatched-spectrum case. Then, the template spectrum is optimized in order to maximize the $\mathrm{CCF}$ peak. During this process, planet atmospheric properties are inferred, including composition, abundance ratio, cloud patchiness, chemical equilibrium, etc. If the optimization process is successful, the CCF $\mathrm{S} / \mathrm{N}$ is limited by the intrinsic structure of the $\mathrm{CCF}$. At this stage, an autocorrelation function is calculated from the optimized template spectrum and subtracted from the optimized $\mathrm{CCF}$ to remove intrinsic structures. After the subtraction, the data reduction and spectral retrieval can potentially reach the photon-noise limit.

\subsubsection{Optimal Band for Planet Detection}

Figure 4 shows CCF S/Ns at star light suppression levels up to $10^{-6}$. At a low level of star light suppression $\left(>10^{-2}\right)$, the $L^{\prime}$ band outperforms other bands because the planet/star contrast is favorable (see Table 2). However, the $L^{\prime}$ curves level off quickly as the star light suppression level increases because sky background becomes the dominant noise source. In this case, increasing star light suppression level does not improve the $\mathrm{CCF} \mathrm{S} / \mathrm{N}$. However, we note that the star light suppression at the beginning of the plateau depends on the brightness of a star. That is, deeper star light suppression is needed to reach the background limit for brighter stars.

At deeper star light suppression, $H$ and $K_{S}$ band become the optimal bands that give the highest CCF S/N. The transition of performance between $L^{\prime}$ and $H / K_{S}$ band takes place at star light suppression levels between $\sim 10^{-1}$ and $10^{-3}$ depending on different cases.

For a given angular separation, there is a trade-off between operating wavelength and wavefront quality. For instance, the Strehl ratio is worse at shorter wavelengths, but spatial resolution improves. Coronagraph performance is usually better with more beam widths $(\lambda / D$ in angle) separating the star and planet. In our simulations, we scale the nominal $10 \%$ throughput with the Strehl ratio to account for better wavefront quality at longer wavelengths, which results in a better coronagraph performance and fiber coupling efficiency. We 


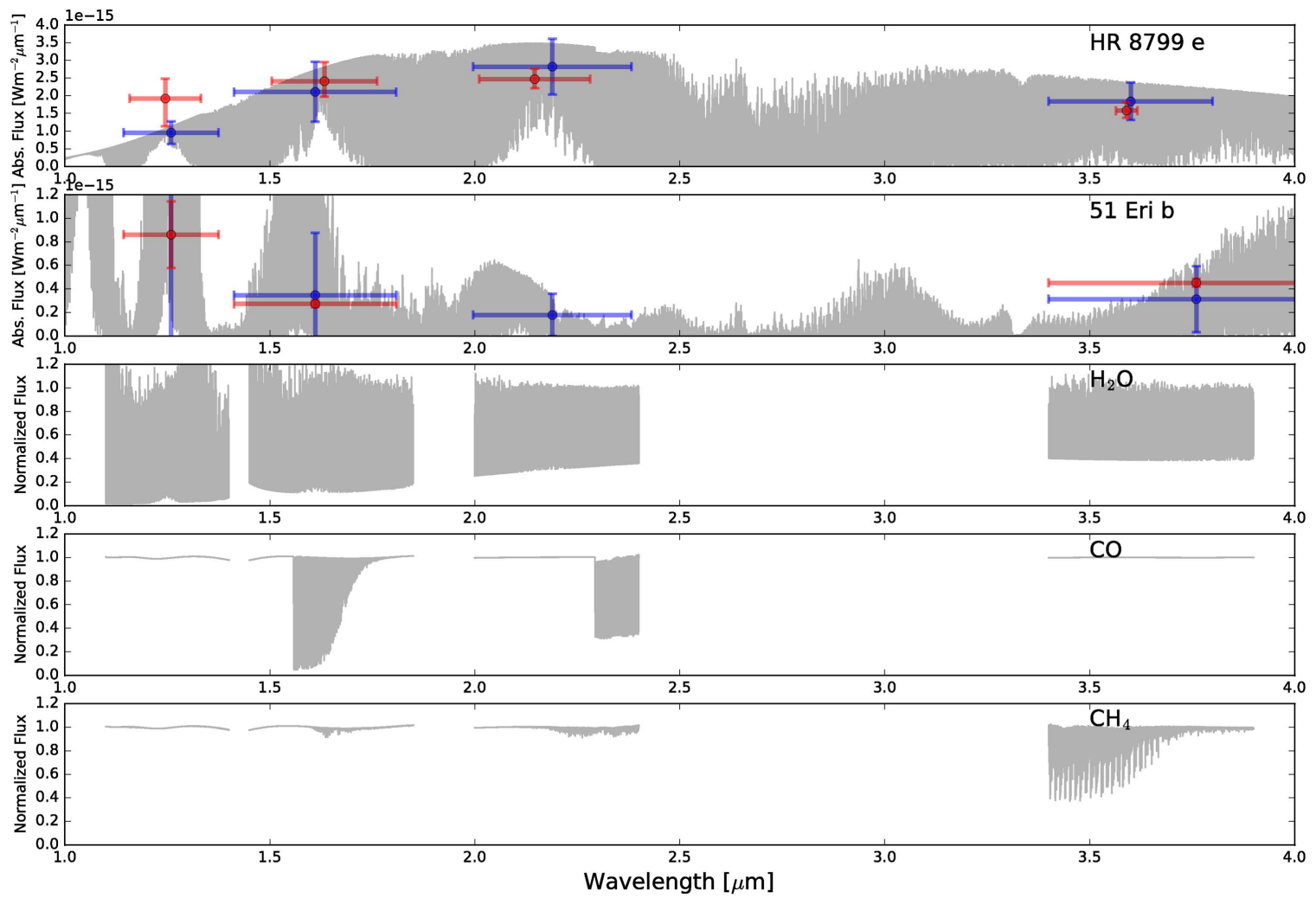

Figure 3. Top two panels: BT-Settl spectra from HR 8799 e and 51 Eri b and comparison of absolute flux between model (blue) and observation (red, Bonnefoy et al. 2016; Macintosh et al. 2015) in different photometric bands. Bottom three panels: normalized spectra for individual molecular species. These spectra are used for the detection of molecular species in the atmosphere of HR 8799 e and 51 Eri b.
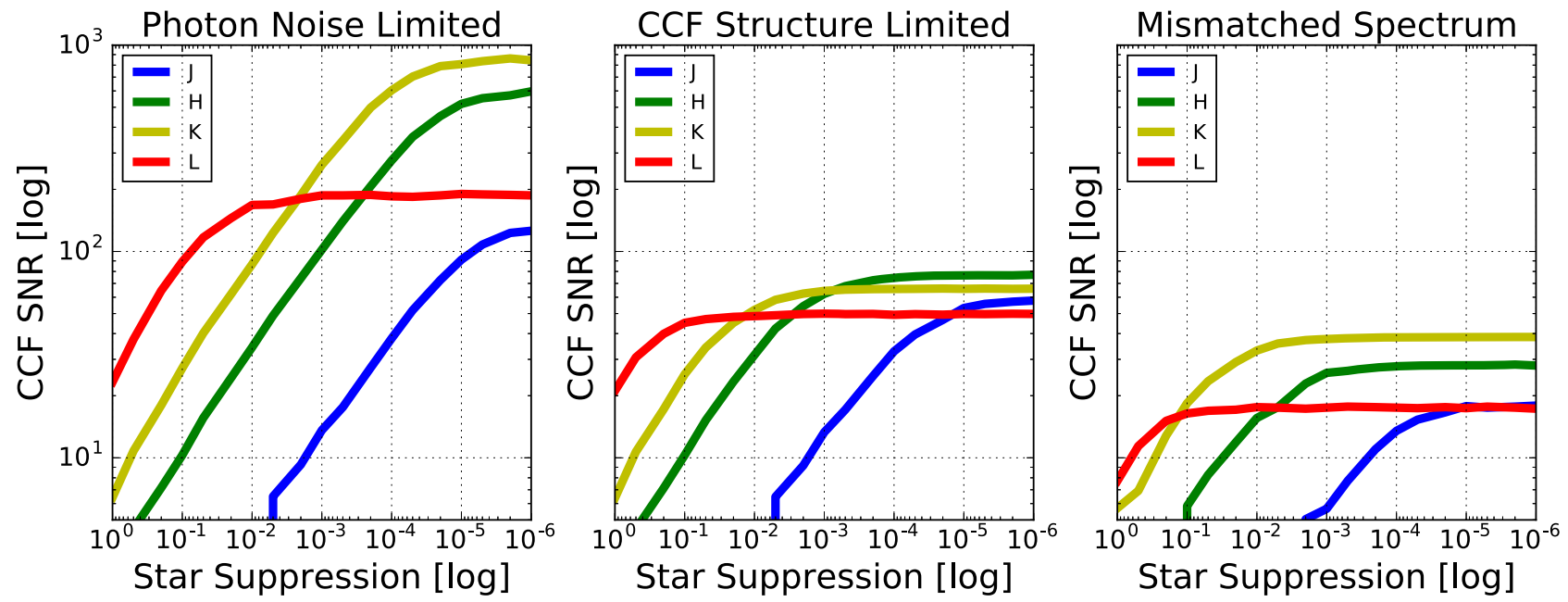

Figure 4. CCF S/N vs. star light suppression level for HR 8799 e in $1 \mathrm{hr}$ exposure time for three cases (see discussion in Section 4.1.1). Simulation parameters for the planet, star, telescope, and instrument are provided in Tables 1 and 2.

do not directly include the benefit of higher resolution at shorter wavelengths because angular separations (in units of $\lambda / D$, see Table 2) for HR 8799 e are much larger than the spatial resolution of KPIC.

\subsubsection{Sensitivity Gain in HDC Observation}

Compared to ground-based HCI observations of HR 8799 e, HDC observations would provide a significant gain in sensitivity. In $L^{\prime}$ band, the detection significance is $5-10$ for HCI only on the Keck telescope (Currie et al. 2014). In comparison, our simulations indicate that at a level of star light suppression of $10^{-3}$, the CCF $\mathrm{S} / \mathrm{N}$ in $L^{\prime}$ is between $\sim 20$ (mismatched-spectrum case) and 200 (photon-noise-limited case). This is a factor of $\sim 2-40$ gain in sensitivity with the help of HRS. The gain is possible because HRS serves as an additional filter for the planet signal. However, the gain in $L^{\prime}$ band is limited by strong sky emission.

In other bands for which the sensitivity is not limited by the sky background but by the planet/star contrast, we expect an HDC instrument to provide an even higher gain in sensitivity. For example, the planet/star contrast for HR 8799 e is 

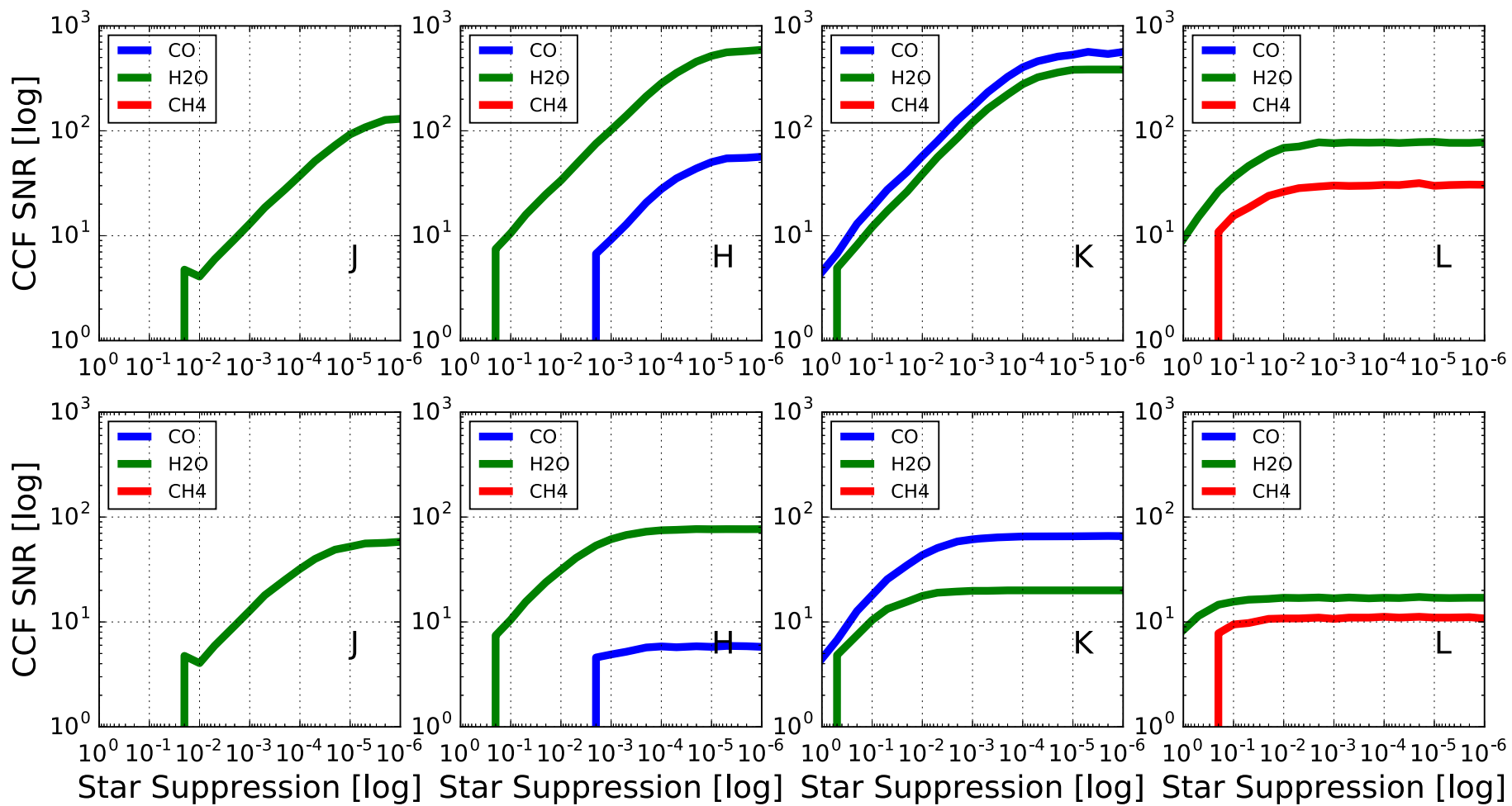

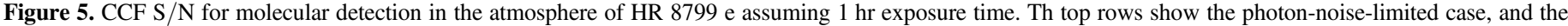
bottom rows show the mismatched-spectrum case. Simulation parameters for the planet, star, telescope, and instrument are provided in Tables 1 and 2.

$\sim 4 \times 10^{-5}$ in $K_{S}$, which may not be seen by an $\mathrm{HCI}$ instrument with star light suppression level of $10^{-3}$. With an HDC instrument, the planet can be detected with a CCF S/N of 40-250 (Figure 4).

\subsubsection{Molecular Detection}

In addition to planet detection using the cross-correlation method, we also consider detecting individual molecular species in the atmosphere of a planet. The template spectrum for a single molecular species is generated as described in Section 2.1.1. In total, we generate spectra for three molecular species: $\mathrm{CO}, \mathrm{H}_{2} \mathrm{O}$, and $\mathrm{CH}_{4}$. They are plotted in Figure 3 along with BT-Settl spectra for HR 8799 e and 51 Eri b.

While $H, K_{S}$, and $L^{\prime}$ bands are identified as the optimal bands for a detection of HR 8799 e, we investigate the potential for using all four bands in searching for molecular species in the atmosphere of HR 8799 e. To do so, we cross-correlate the simulated observed planet spectrum with a template spectrum of an individual molecular species.

Figure 5 shows the $\mathrm{CCF} \mathrm{S} / \mathrm{N}$ as a function of star light suppression level for $\mathrm{CO}, \mathrm{H}_{2} \mathrm{O}$, and $\mathrm{CH}_{4}$ for $\mathrm{J}_{-}, \mathrm{H}_{-}, \mathrm{K}_{\mathrm{S}^{-}}$, and $L^{\prime}$-band observations. The optimal bands for detections of $\mathrm{CO}$, $\mathrm{H}_{2} \mathrm{O}$, and $\mathrm{CH}_{4}$ are $K_{S}, H$, and $L^{\prime}$, respectively. The differences between the optimal bands for planet detection and molecular species detection highlights the need for multiband highresolution spectroscopy.

When we compare our results to previous studies, our finding in $K_{S}$ band is consistent with Keck OSIRIS observations of HR $8799 \mathrm{c}$. Planet c has a similar effective temperature and surface temperature as HR 8799 e. With a star light suppression level of $\sim 10^{-2}$, Konopacky et al. (2013) detected $\mathrm{CO}$ and $\mathrm{H}_{2} \mathrm{O}$ in HR 8799 c with Keck OSIRIS at a CCF S/N of $\sim 10$. That the CCF $\mathrm{S} / \mathrm{N}$ is lower than predicted in Figure 5 can be attributed to the lower spectral resolution and higher detector noise.

The sharp drop of the CCF S/N at low levels of star light suppression in all subplots of Figure 5 is due to the criteria for planet/molecular detection in our simulation. In order to be qualified as a significant detection, we require that (1) the CCF $\mathrm{S} / \mathrm{N}$ is higher than 3 and (2) the RV of CCF peak is consistent with the input planet RV within one resolution element. Without the second criterion, there may be interlopers from random $\mathrm{CCF}$ fluctuation due to noise that may be misinterpreted as $\mathrm{CCF}$ peaks. In practice, the measured CCF RVs should also follow a pattern that is consistent with the planet orbits. Therefore, if $>50 \%$ of the simulations result in an inconsistent $\mathrm{RV}$, we assign a zero value to $\mathrm{CCF} S / \mathrm{N}$. The result implies that the minimum $\mathrm{CCF} S / \mathrm{N}$ is $\sim 10$ to confirm that the absorption or emission signal truly originates from the planet.

\subsection{Eri $b$}

Planet 51 Eri b (Macintosh et al. 2015) is the only directly imaged planet whose inferred mass is within the planet mass regime according to both cold-start and hot-start models (Bowler 2016). Furthermore, its brightness contrast and angular separation are representative of the practical detection limits of current ground-based high-contrast imagers. We therefore simulate observations of 51 Eri b with an HDC instrument to provide a point of comparison with the current state-of-the-art modeling.

The input parameters for the planet, host star, telescope, and instrument are provided in Tables 1 and 3. We use input spectra with $T_{\text {eff }}=700 \mathrm{~K}$ and $\log (g)=3.5$ for the planet and $T_{\text {eff }}=7400 \mathrm{~K}$ and $\log (g)=4.0$ for the star. The metallicity $[\mathrm{Fe} / \mathrm{H}]$ is set to zero for both planet and star. 
Table 3

51 Eri and Planet b

\begin{tabular}{|c|c|c|c|}
\hline Parameter & Value & Unit & References \\
\hline \multicolumn{4}{|l|}{$\overline{\text { Star }}$} \\
\hline $\begin{array}{l}\text { Effective temperature } \\
\qquad\left(T_{\text {eff }}\right)^{\mathrm{a}}\end{array}$ & 7400 & $\mathrm{~K}$ & $\cdots$ \\
\hline Surface gravity $(\log g)^{\mathrm{a}}$ & 4.0 & cgs & $\cdots$ \\
\hline Distance & 29.40 & $\mathrm{pc}$ & $\begin{array}{l}\text { Macintosh } \\
\text { et al. (2014) }\end{array}$ \\
\hline Rotational velocity & 50.0 & $\mathrm{~km} \mathrm{~s}^{-1}$ & $\ldots$ \\
\hline Inclination $(i)$ & 40.0 & degree & $\cdots$ \\
\hline Radial velocity & -12.6 & $\mathrm{~km} \mathrm{~s}^{-1}$ & Gontcharov (2006) \\
\hline \multicolumn{4}{|l|}{ Planet } \\
\hline $\begin{array}{l}\text { Effective temper- } \\
\quad \text { ature }\left(T_{\text {eff }}\right)\end{array}$ & $550-750$ & $\mathrm{~K}$ & $\begin{array}{l}\text { Macintosh } \\
\text { et al. (2014) }\end{array}$ \\
\hline Surface gravity $(\log g$ ) & 3.5 & $\operatorname{cgs}$ & $\begin{array}{l}\text { Macintosh } \\
\text { et al. (2014) }\end{array}$ \\
\hline Rotational velocity & 15.0 & $\mathrm{~km} \mathrm{~s}^{-1}$ & $\ldots$ \\
\hline Inclination $(i)$ & 45 & degree & $\cdots$ \\
\hline Projected separation (a) & 13.2 & $\mathrm{au}$ & $\begin{array}{l}\text { Macintosh } \\
\text { et al. (2014) }\end{array}$ \\
\hline Radial velocity $^{\mathrm{b}}$ & -12.6 & $\mathrm{~km} \mathrm{~s}^{-1}$ & Gontcharov (2006) \\
\hline Angular separation & 0.45 & $\operatorname{arcsec}$ & $\begin{array}{l}\text { Macintosh } \\
\text { et al. (2014) }\end{array}$ \\
\hline Angular separation in $J$ & 17.3 & $\lambda / D$ & $\begin{array}{l}\text { Macintosh } \\
\text { et al. (2014) }\end{array}$ \\
\hline Angular separation in $H$ & 13.5 & $\lambda / D$ & $\begin{array}{l}\text { Macintosh } \\
\text { et al. (2014) }\end{array}$ \\
\hline Angular separation in $K_{S}$ & 9.9 & $\lambda / D$ & $\begin{array}{l}\text { Macintosh } \\
\text { et al. (2014) }\end{array}$ \\
\hline Angular separation in $L^{\prime}$ & 5.8 & $\lambda / D$ & $\begin{array}{l}\text { Macintosh } \\
\text { et al. (2014) }\end{array}$ \\
\hline Planet/star contrast in $J$ & $2.6 \times 10^{-6}$ & $\ldots$ & $\ldots$ \\
\hline Planet/star contrast in $H$ & $1.1 \times 10^{-6}$ & $\cdots$ & $\ldots$ \\
\hline Planet/star contrast in $K_{S}$ & $1.7 \times 10^{-6}$ & $\ldots$ & $\ldots$ \\
\hline Planet/star contrast in $L^{\prime}$ & $2.7 \times 10^{-5}$ & $\cdots$ & $\ldots$ \\
\hline
\end{tabular}

Notes.

a Based on F0IV spectral estimation from Macintosh et al. (2014).

b Assumed to be the same as 51 Eri.

We adjust the planet and star flux such that the model flux and the absolute flux measured from photometry are consistent within the uncertainties (Figure 3). We adopt values from Macintosh et al. (2015) for the absolute flux measurement. Similar to the cross-correlation calculation presented for HR 8799 e, we use the BT-Settl spectrum as input to simulate observations. For the template spectrum used for the crosscorrelation, we either use the same spectrum as the input planet spectrum or the combined molecule-by-molecule spectrum of $\mathrm{CO}, \mathrm{CH}_{4}$, and $\mathrm{H}_{2} \mathrm{O}$.

\subsubsection{Optimal Band for Planet Detection}

Figure 6 shows the CCF $\mathrm{S} / \mathrm{N}$ for 51 Eri $\mathrm{b}$ in three cases. We again observe a decreasing trend of the CCF $\mathrm{S} / \mathrm{N}$ from the photon-noise-limited case to the cases dominated by systematics. $J$ and $L^{\prime}$ bands are optimal bands for detecting 51 Eri b. The $L^{\prime}$-band observation yields the highest CCF $\mathrm{S} / \mathrm{N}$ for the photon-noise-limited case and the CCF structure-limited case at low star light suppression levels $\left(>10^{-3}\right)$. However, the planet cannot be detected in $L^{\prime}$ band in the mismatched-spectrum case. This is possibly because of a poor knowledge of the $L^{\prime}$-band planet spectrum. $J$ band is the optimal band for the photonnoise-limited case and the CCF structure-limited case if the star light suppression levels are better than a few times $10^{-4}$. In addition, $J$ band is also the optimal band for the mismatchedspectrum case. This is largely due to the high photon flux from the planet in $J$ band.

In the photon-noise-limited case and the CCF structurelimited case, we use a planet template spectrum that is exactly the same as the planet spectrum used in simulating the observation. This is to assume that we have full knowledge of the planet spectrum. While this assumption leads to a much higher CCF S/N (see Figure 6), we cannot practically generate a perfect planet or molecular template spectrum.

To demonstrate this point, we use the BT-Settl spectrum as an input to simulate the astrophysical signal. We use the combined molecular spectrum for $\mathrm{CO}, \mathrm{H}_{2} \mathrm{O}$, and $\mathrm{CH}_{4}$ as the template spectrum. As a result, the $\mathrm{CCF} \mathrm{S/N}$ is reduced for all bands (see Figure 6). Using an imperfect template in the crosscorrelation operation may even lead to missed detections of planets or particular molecular species. However, as mentioned in Section 4.1.1, the mismatched-spectrum case also represents an opportunity for atmospheric retrieval.

\subsubsection{Molecular Detection}

Figure 7 shows the CCF S/N achieved by cross-correlating the reduced spectrum with template spectrum of individual molecular species. Depending on the photon flux and the density and strength of the spectral lines, the optimal band is different for each species. $\mathrm{H}_{2} \mathrm{O}$ is present in all $J, H, K_{S}$, and $L^{\prime}$ bands (see Figure 3 ) and can be detected in $J, H$, and $K_{S}$ band. The highest CCF S/N is given in $J$ band. CO has lines in $H$ and $K_{S}$ band and can be detected in $H$ band. Although abundant $\mathrm{CH}_{4}$ lines exist in $L^{\prime}$ band, $\mathrm{CH}_{4}$ in 51 Eri b cannot be detected because the sky background is much higher and the photon flux is much lower than for HR 8799 e.

\section{Searching and Characterizing Earth-like Planets around Low-mass Stars with Ground-based Extremely Large Telescopes}

Searching for Earth-like planets and identifying molecular species in their atmospheres is one of the main science goals for ground-based extremely large telescopes and future spacebased missions. Ground-based telescopes are generally larger than space-based telescopes and thus have the advantage of higher angular resolution at a given wavelength. On the other hand, space-based telescopes can achieve deeper star light suppression than ground-based instruments because of their vantage point outside our turbulent atmosphere. These differences in spatial resolution and achievable contrast levels affect the science objectives of space-based and ground-based missions for the study of Earth-like planets. Ground-based missions are more suitable in studying Earth-like planets around low-mass stars because (1) the requirements for star light suppression are less stringent and (2) the IWA may be improved by the increased telescope aperture size. In comparison, space-based missions are better for targeting Earth-like planets around solar-type stars because (1) the star light is more strongly suppressed and (2) the requirements for IWA are less stringent.

The recent discovery of Proxima Cen b (Anglada-Escudé et al. 2016) makes this Earth-like planet candidate an excellent target to characterize. However, this requires significantly upgraded capabilities of current telescopes (Lovis et al. 2017). 

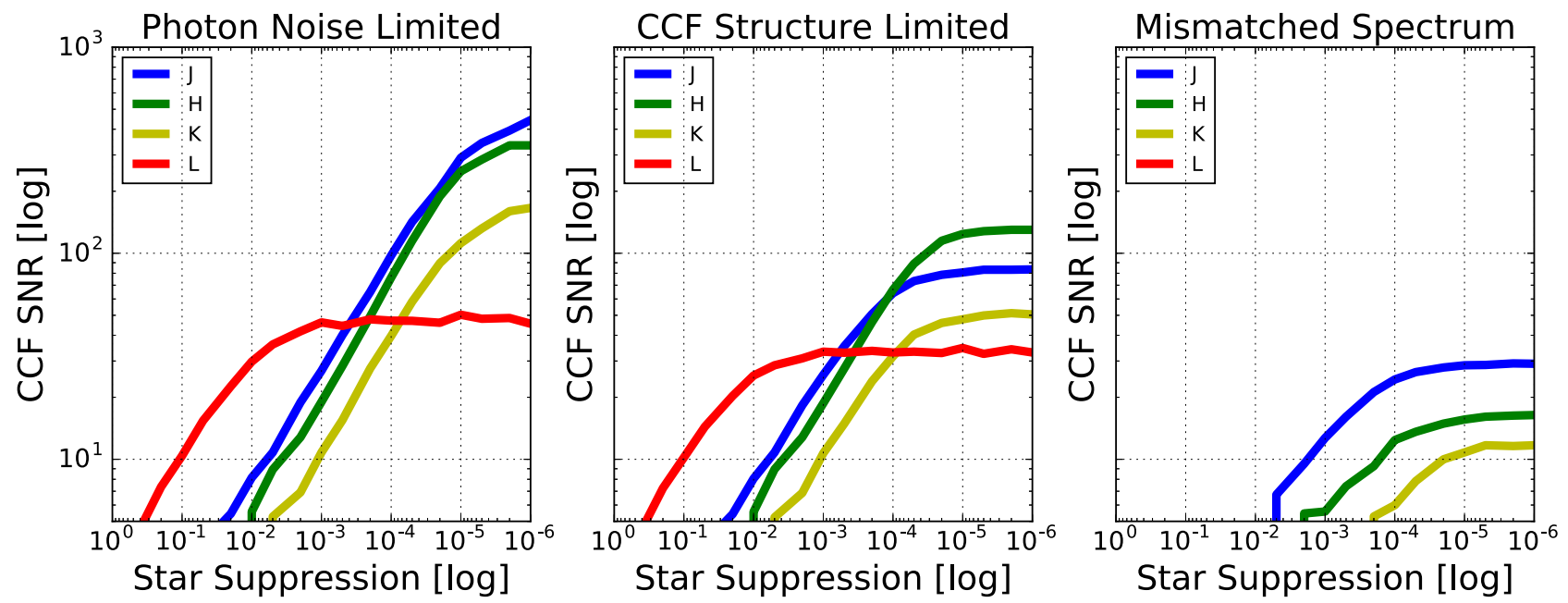

Figure 6. $\mathrm{CCF}$ S/N vs. star light suppression level for $51 \mathrm{Eri} \mathrm{b}$ in $1 \mathrm{hr}$ exposure time for three cases. Simulation parameters for the planet, star, telescope, and instrument are provided in Tables 1 and 3.
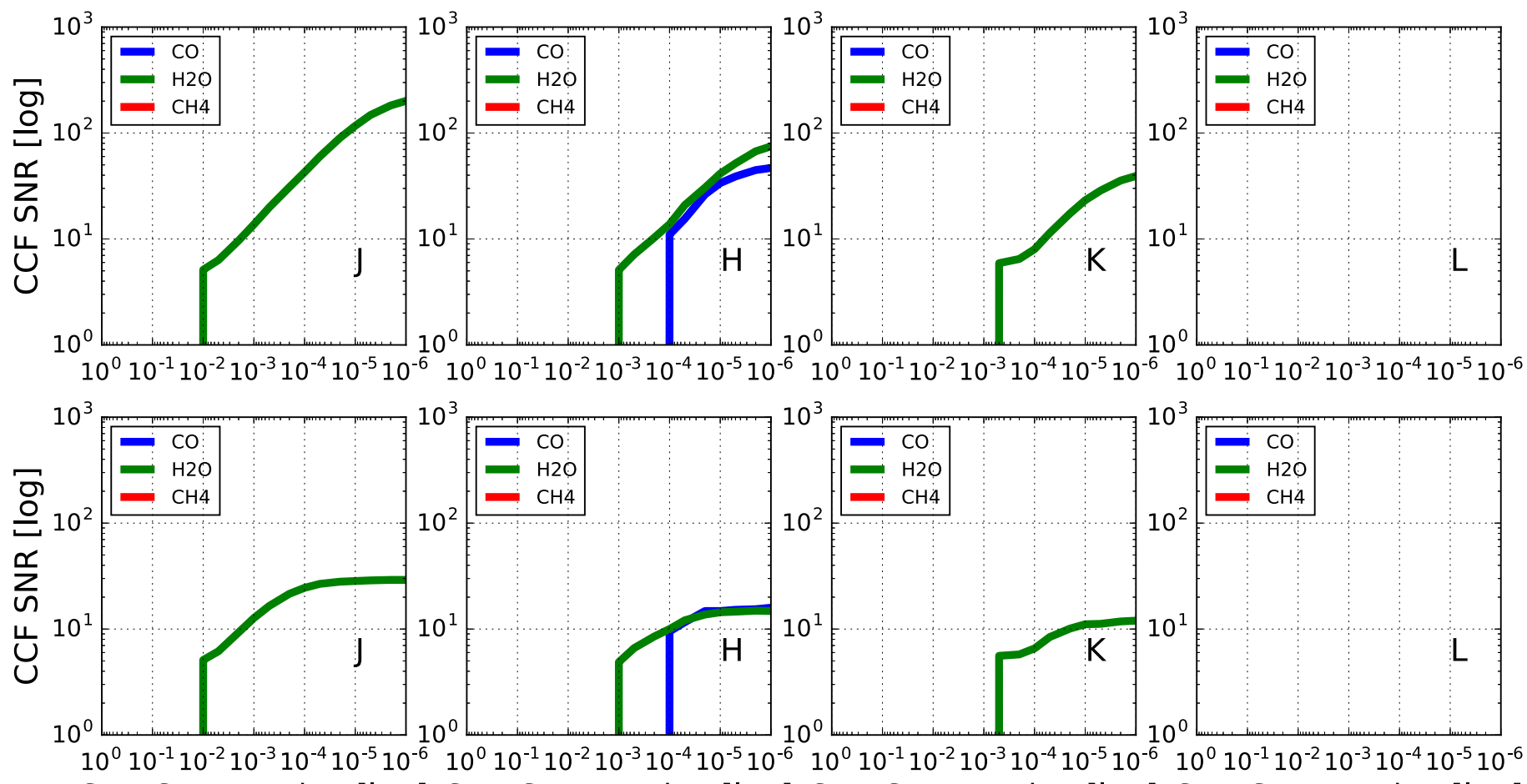

Star Suppression [log] Star Suppression [log] Star Suppression [log] Star Suppression [log]

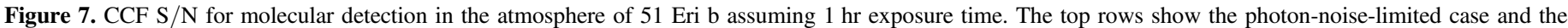
bottom rows show the mismatched-spectrum case. Simulation parameters for the planet, star, telescope, and instrument are provided in Tables 1 and 3.

To demonstrate the potential of HDC for a $30 \mathrm{~m}$ class telescope, we simulate observations of (1) Proxima Cen b and (2) an Earth-like planet in the habitable zone of an M dwarf at $5 \mathrm{pc}$. The second case represents a general case study, whereas the first is the best-case scenario owing to the proximity of Proxima Cen.

We simulate observations in $J, H, K_{S}$ and $L^{\prime}$ band and find that the CCF S/N in $L^{\prime}$ does not reach the detection threshold within the considered star light suppression levels and spectral resolutions, therefore we only discuss $J, H$, and $K_{S}$ results here. While $L^{\prime}$ band is not an optimal band to search for planets in reflected light, longer wavelengths (e.g., $M$ and $N$ band) may be considered in the search for planet emission. Bandwidths for $J$ and $K_{S}$ bands are within $20 \%$ and $H$ band is $\sim 25 \%$. While it is challenging to maintain a consistent star light suppression level over such a wide bandwidth, we consider the full wavelength range for these bands. In practice, suboptimal wide-band performance may be improved by multiple observations with narrower bandwidths.

\subsection{Simulation Setup}

We use the Earth albedo spectrum (Figure 8) for the planet, which is a product of the stellar spectrum and the albedo spectrum. The absolute flux of the spectrum is then scaled with the planet radius, the planet-star separation, and the planet 

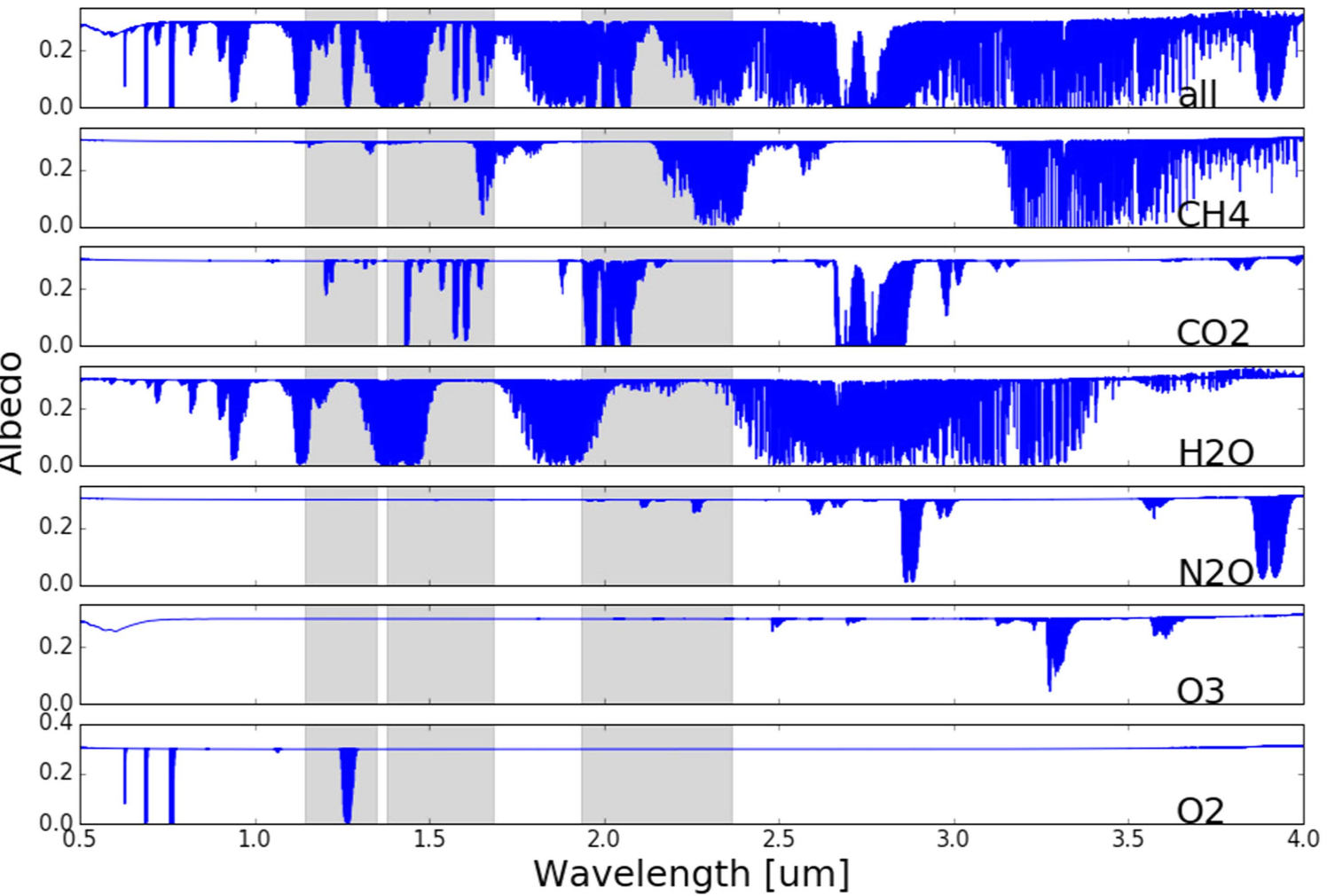

Figure 8. Albedo spectrum of an Earth-like planet. We consider the average albedo between a high-cloud case (high albedo) and cloud-free case (low albedo). Shaded regions are wavelength regions we consider to simulate observations for detecting molecular species with ground-based telescopes. For space-based observation, we consider a wavelength region from 0.5 to $1.7 \mu \mathrm{m}$.

fractional illuminated area (i.e., phase function). We use the BT-Settl spectrum with $T_{\text {eff }}=3500 \mathrm{~K}$ and $\log (g)=4.5$ as the input M-dwarf spectrum. For Proxima Cen, we use the BT-Settl spectrum with $T_{\text {eff }}=3000 \mathrm{~K}$ and $\log (g)=5.0$. The metallicity $[\mathrm{Fe} / \mathrm{H}]$ is set to zero for all cases. The telescope and instrument parameters used in simulation can be found in Table 4. Further information about the planet and star can be found in Tables 5 and 6.

\subsection{Results for $J$, $H$, and $K_{S}$ Bands \\ 5.2.1. An Interplay Between HCI and HRS}

Figures 9-11 show the CCF $\mathrm{S} / \mathrm{N}$ contours as a function of spectral resolution and star light suppression level for $J$-, $H$-, and $K_{S}$-band observations. The general trend is that the CCF S/N increases with higher spectral resolution and deeper levels of star light suppression. As a result, high spectral resolution relaxes the star light suppression requirements by several orders of magnitude. This has significant implications for HDC observations: insufficient star light suppression may be compensated for by increasing spectral resolution.

The planet/star contrast is $\sim 10^{-8}-10^{-7}$ for the M-dwarf planet and Proxima Cen b systems. However, it is extremely challenging to achieve a star light suppression level of $\sim 10^{-8}$ from the ground. With the help of HRS, the star light suppression requirement can be relaxed by about 2-3 orders of magnitude. While there is no clear pathway to achieve $\sim 10^{-8}$ star light suppression levels with ground-based telescopes, $10^{-5}-10^{-6}$ is a much more attainable goal, which is within reach of mainstream extreme $\mathrm{AO}$ systems currently operating on most $8-10 \mathrm{~m}$ class telescopes.
Table 4

Telescope and Instrument Parameters for M-dwarf Planets (Proxima Cen b and an M-dwarf Planet System at $5 \mathrm{pc}$ )

\begin{tabular}{lcc}
\hline \hline Parameter & Value & Unit \\
\hline Telescope aperture & 30.0 & $\mathrm{~m}$ \\
Telescope+instrument throughput & $10 \%$ & $\ldots$ \\
Wavefront correction error floor & 200 & $\mathrm{~nm}$ \\
Spectral resolution & varied & $\ldots$ \\
$J$-band spectral range & $1.143-1.375$ & $\mu \mathrm{m}$ \\
$H$-band spectral range & $1.413-1.808$ & $\mu \mathrm{m}$ \\
$K$-band spectral range & $1.996-2.382$ & $\mu \mathrm{m}$ \\
Exposure time & 100 & $\mathrm{hour}$ \\
Fiber angular diameter & 1.0 & $\lambda / D$ \\
Readout noise & 0.0 or 2.0 & $e^{-\mathrm{a}}$ \\
Dark current & 0.0 or 0.002 & $e^{-} \mathrm{s}^{-1 \mathrm{a}}$ \\
\hline
\end{tabular}

Note.

${ }^{a}$ Based on the H2RG detector specification (Blank et al. 2012).

\subsubsection{Star Light Suppression versus Planet Signal}

Having a larger telescope aperture not only improves angular resolution, but is also critical for gathering sufficient signal. The improved signal increases the CCF S/N, thereby relaxing the requirements for star light suppression. Likewise, star light suppression requirements may be further relaxed by increasing signal via longer exposure times or improving instrument throughput. In all cases, the boost in sensitivity provided by an HDC instrument depends on how much signal the instrument receives and how it compares with the relevant noise sources.

Figure 12 shows the noise sources for the case of a $30 \mathrm{~m}$ telescope observing an M-dwarf planet in the $K_{S}$ band. The plot 
Table 5

Proxima Centauri b Planet System

\begin{tabular}{|c|c|c|}
\hline Parameter & Value & Unit \\
\hline \multicolumn{3}{|l|}{ Star } \\
\hline Effective temperature $^{\mathrm{a}}\left(T_{\text {eff }}\right)$ & 3050 & K \\
\hline Mass & 0.12 & $M_{\odot}$ \\
\hline Radius & 0.14 & $R_{\odot}$ \\
\hline Surface gravity $(\log g)$ & 5.0 & $\operatorname{cgs}$ \\
\hline Metallicity $([M / H])$ & 0.0 & $\operatorname{dex}$ \\
\hline Distance & 1.295 & $\mathrm{pc}$ \\
\hline$V \sin i$ & $<1$ & $\mathrm{~km} \mathrm{~s}^{-1}$ \\
\hline Inclination $(i)$ & 20 & degree \\
\hline Radial velocity & -22.4 & $\mathrm{~km} \mathrm{~s}^{-1}$ \\
\hline \multicolumn{3}{|l|}{ Planet } \\
\hline Effective temperature $\left(T_{\text {eff }}\right)$ & 234 & $\mathrm{~K}$ \\
\hline$V \sin i^{\mathrm{b}}$ & 0.014 & $\mathrm{~km} \mathrm{~s}^{-1}$ \\
\hline Inclination $(i)$ & 20 & degree \\
\hline Semimajor axis $(a)$ & 0.05 & $\mathrm{au}$ \\
\hline Radius & 1.0 & $R_{\oplus}$ \\
\hline Radial velocity & 22.2 & $\mathrm{~km} \mathrm{~s}^{-1}$ \\
\hline Illuminated area & 0.5 & $\cdots$ \\
\hline Planet/star contrast & $1.6 \times 10^{-7}$ & $\cdots$ \\
\hline Angular separation & 38.6 & mas \\
\hline Angular separation in $J$ & 4.5 & $\lambda / D$ \\
\hline Angular separation in $H$ & 3.5 & $\lambda / D$ \\
\hline Angular separation in $K_{S}$ & 2.6 & $\lambda / D$ \\
\hline
\end{tabular}

Notes.

a All values are from Anglada-Escudé et al. (2016). We use $3000 \mathrm{~K}$ in the simulation.

${ }^{\mathrm{b}}$ We assume that the planet is tidally locked.

Table 6

An M Dwarf and an Earth-like Planet

\begin{tabular}{|c|c|c|}
\hline Parameter & Value & Unit \\
\hline \multicolumn{3}{|l|}{ Star } \\
\hline Effective temperature $\left(T_{\text {eff }}\right)$ & 3500 & $\mathrm{~K}$ \\
\hline Mass & 0.5 & $M_{\odot}$ \\
\hline Radius & 0.5 & $R_{\odot}$ \\
\hline Surface gravity $(\log g)$ & 4.5 & $\operatorname{cgs}$ \\
\hline Metallicity $([M / H])$ & 0.0 & $\operatorname{dex}$ \\
\hline Distance & 5.0 & $\mathrm{pc}$ \\
\hline$V \sin i$ & 2.7 & $\mathrm{~km} \mathrm{~s}^{-1}$ \\
\hline Inclination $(i)$ & 20 & degree \\
\hline Radial velocity & 15.0 & $\mathrm{~km} \mathrm{~s}^{-1}$ \\
\hline \multicolumn{3}{|l|}{ Planet } \\
\hline Effective temperature ( $\left.T_{\text {eff }}\right)$ & 300 & $\mathrm{~K}$ \\
\hline Surface gravity $(\log g)$ & 3.0 & $\operatorname{cgs}$ \\
\hline$V \sin i$ & 0.017 & $\mathrm{~km} \mathrm{~s}^{-1}$ \\
\hline Inclination $(i)$ & 20 & degree \\
\hline Semimajor axis $(a)$ & 0.1 & $\mathrm{au}$ \\
\hline Radius & 1.0 & $R_{\oplus}$ \\
\hline Radial velocity & 20.0 & $\mathrm{~km} \mathrm{~s}^{-1}$ \\
\hline Illuminated area & 0.5 & $\cdots$ \\
\hline Planet/star contrast & $6.2 \times 10^{-9}$ & $\cdots$ \\
\hline Angular separation & 20.0 & mas \\
\hline Angular separation in $J$ & 2.3 & $\lambda / D$ \\
\hline Angular separation in $H$ & 1.8 & $\lambda / D$ \\
\hline Angular separation in $K_{S}$ & 1.3 & $\lambda / D$ \\
\hline
\end{tabular}

compares noise sources as a function of spectral resolution. Depending on the spectral resolution and star light suppression level, the dominating noise source can be sky background, photon noise, and detector noise. For example, sky background noise dominates at deep star light suppression levels and low spectral resolution. Photon noise from the leaked stellar light dominates at low level of star light suppression. Detector noise dominates at deep star light suppression and high spectral resolution.

Ground-based HDC instruments usually operate at high spectral resolution $(R \sim 100,000)$ and a star light suppression level that is a few orders of magnitude lower than the planet/ star contrast. Therefore the photon noise from the leaked star light typically is the dominant noise source. The detector and sky background noise sources are $\sim 5$ times lower than the photon noise source at a $10^{-6}$ star light suppression level. The $\mathrm{S} / \mathrm{N}$ per pixel is on the order of unity or lower in the high spectral resolution regime. In order to achieve higher sensitivity at lower levels of star light suppression, increasing the signal from the planet is the key.

\subsubsection{Photon-noise-limited versus CCF Structure-limited Case}

The solid and dashed contours in Figures 9-11 represent two different cases: the photon-noise-limited case and the CCF structure-limited case. In the high spectral resolution regime, the two contours usually agree with each other. The agreement between two sets of contours can be explained by the low $\mathrm{S} / \mathrm{N}$ per pixel in the high spectral resolution regime. In the situation of a low $\mathrm{S} / \mathrm{N}$ per pixel, the $\mathrm{CCF}$ fluctuation is mainly due to photon noise. Therefore the $\mathrm{CCF} S / \mathrm{N}$ in the the photon-noiselimited case is essentially the same case as the CCF structurelimited case. In contrast, in the low spectral resolution regime, the $\mathrm{S} / \mathrm{N}$ per pixel is higher, as shown by Figure 12 . The $\mathrm{CCF}$ fluctuation is no longer due to photon noise, but to intrinsic $\mathrm{CCF}$ structures. Therefore the higher $\mathrm{S} / \mathrm{N}$ per pixel causes the $\mathrm{CCF} \mathrm{S/N}$ in the two cases to deviate from each other. This is true for the simulations in all bands. We observe similar deviations in our simulations for HR 8799 e (Section 4.1) and 51 Eri b (Section 4.2).

\subsubsection{Molecular Detection}

If Earth-sized planets around $\mathrm{M}$ dwarfs have atmospheres similar to the Earth's, $\mathrm{H}_{2} \mathrm{O}, \mathrm{O}_{2}, \mathrm{CO}_{2}$, and $\mathrm{CH}_{4}$ may potentially be detected with HDC instruments on $30 \mathrm{~m}$ class telescopes. On the other hand, $\mathrm{N}_{2} \mathrm{O}$ and $\mathrm{O}_{3}$ are not detectable because of their lack of lines in the considered wavelength range. $\mathrm{O}_{2}$ can only be detected in the $J$ band, $\mathrm{H}_{2} \mathrm{O}$ is detectable in the $J$ and $H$ bands. Searching for $\mathrm{CO}_{2}$ and $\mathrm{CH}_{4}$ is better conducted in the $K_{S}$ band because of the molecule line density and depth. For the M-dwarf planet case, $\mathrm{CH}_{4}$ cannot be detected in the $H$ band with a CCF $\mathrm{S} / \mathrm{N}$ over 10 .

\section{Searching and Characterizing Earth-like Planets around Solar-type Stars with Space-based Telescopes}

Space-based instruments may achieve deep star light suppression $\left(<10^{-8}\right)$ and will therefore allow observations that would be extremely challenging from the ground; i.e., observing a Sun-Earth system for which the planet/star contrast is $\sim 10^{-10}$. In addition, space-based observations are free from contamination due to the Earth's atmosphere that may cause confusion when detecting molecular species that exists in both the Earth's and the exoplanet's atmosphere. However, telescope apertures for space-based observations are typically much smaller than ground-based facilities. 

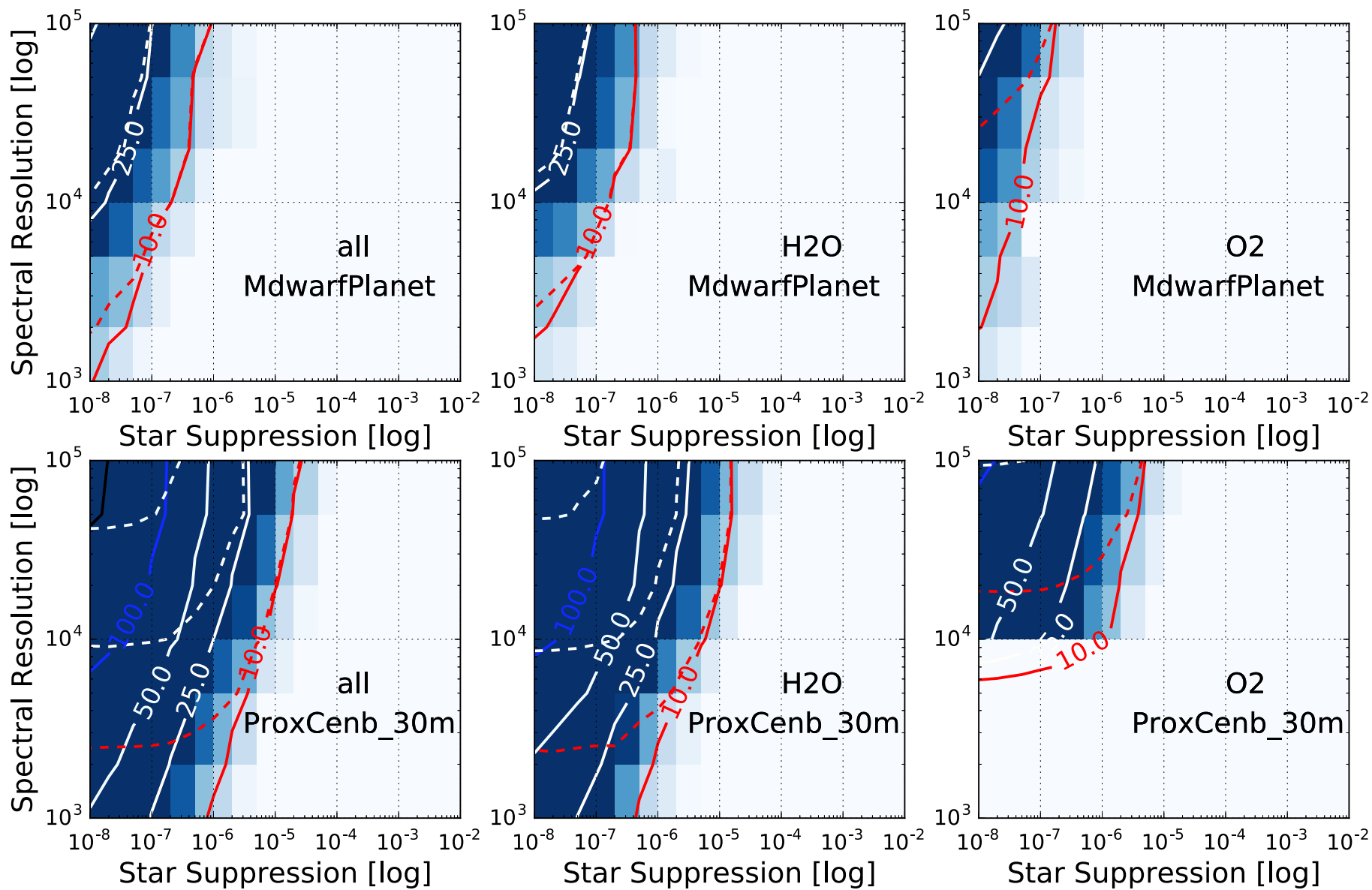

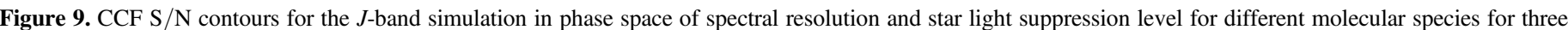

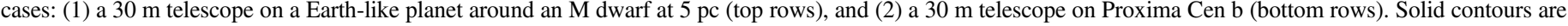

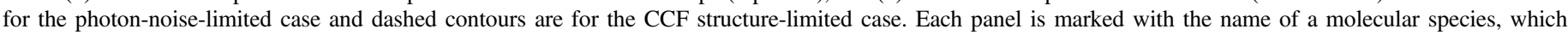
indicates that only the lines of a given molecular species are used in the cross-correlation. "All" means that all lines are used.
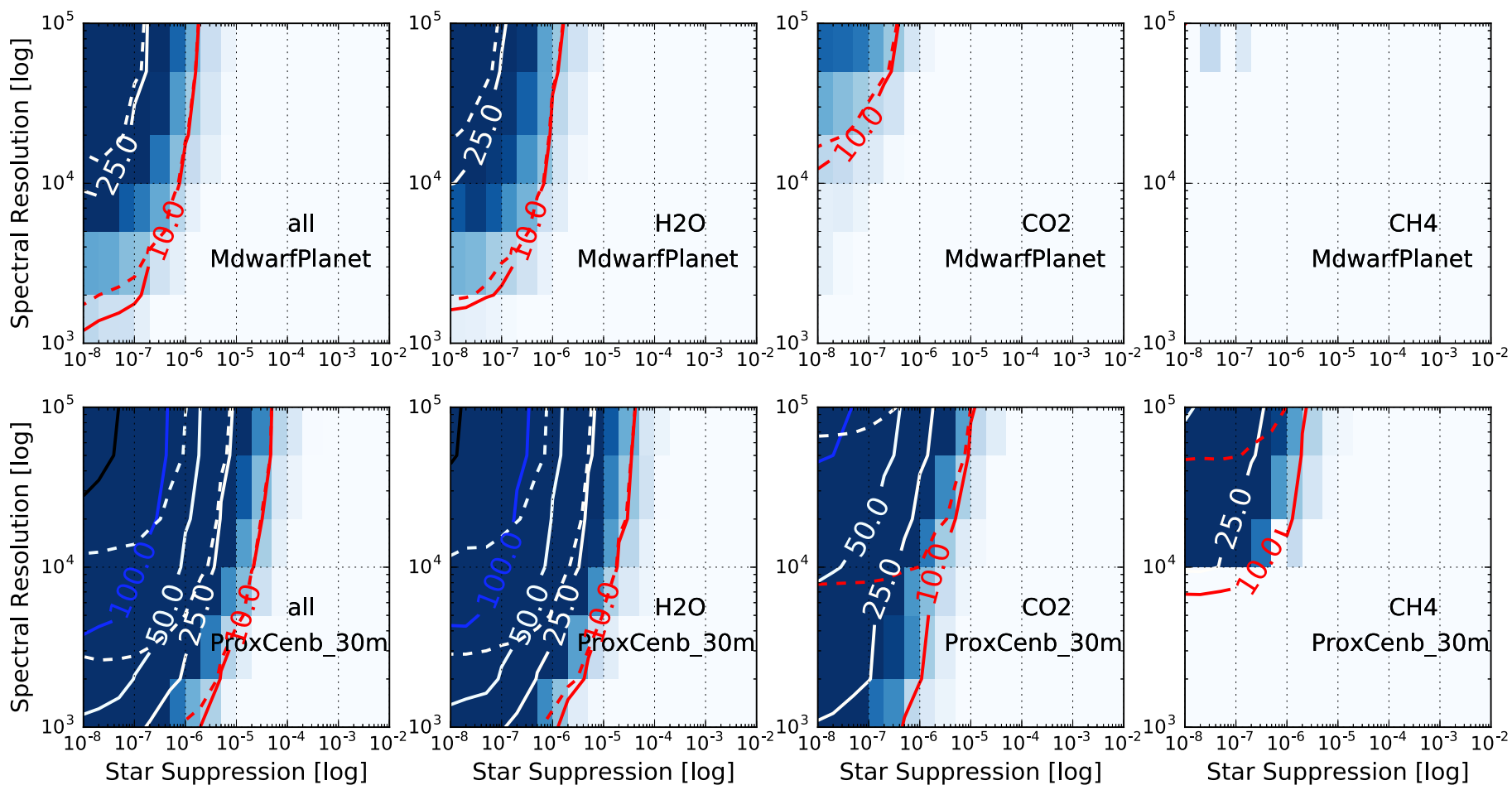

Figure 10. Same as Figure 9, but for the $H$-band simulation. 

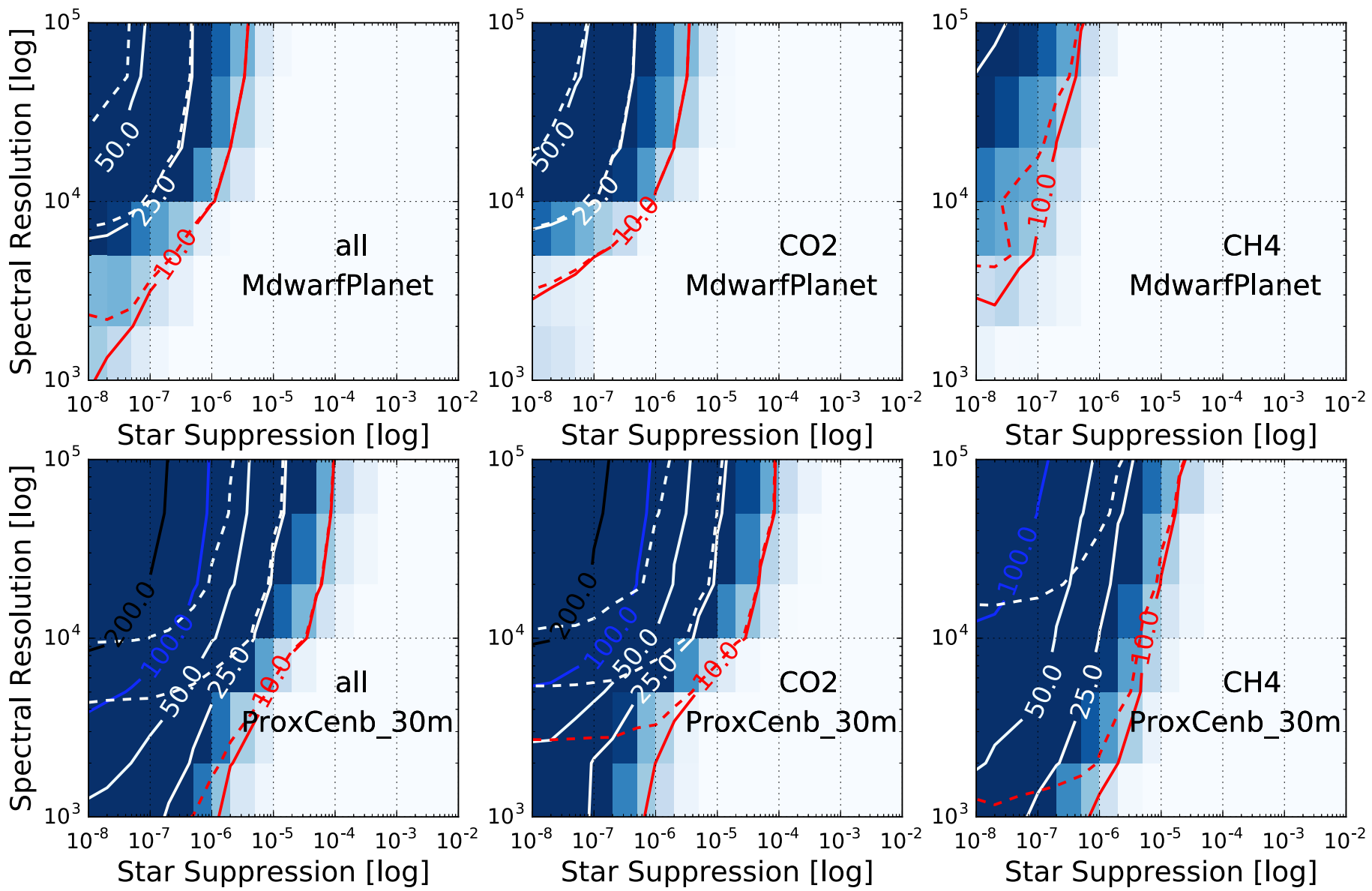

Figure 11. Same as Figure 9 , but for the $K_{S}$-band simulation.

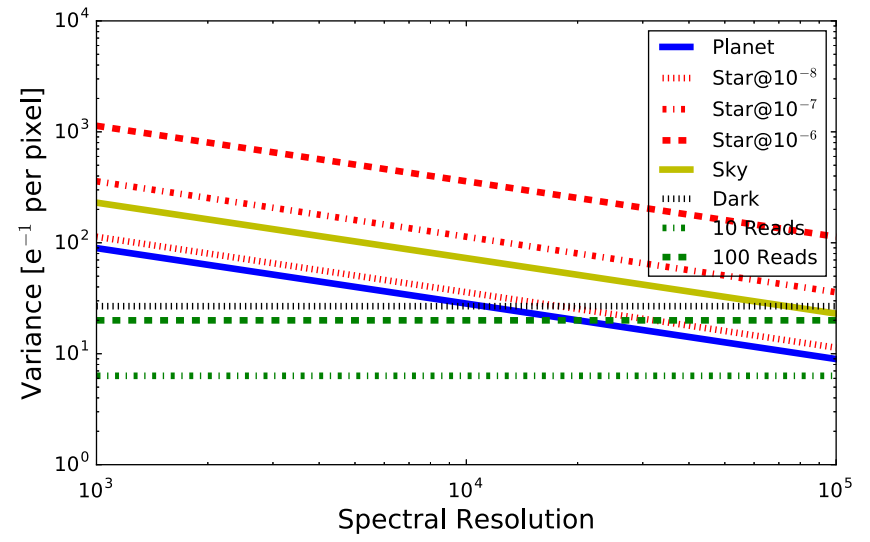

Figure 12. Comparison of noise sources at different spectral resolutions for the case of a $30 \mathrm{~m}$ telescope on an M-dwarf planet in $K_{S}$ band.

Next-generation space-based telescopes for high-contrast observations will range from 4 to $16 \mathrm{~m}$ in diameter. The angular separation of a Sun-Earth system at $5 \mathrm{pc}(0$ ". 2$)$ would be 2.4-7.3 $\lambda / D$ in $H$ band and 5.2-15.5 $\lambda / D$ in $r$ band, which is within the working angle range of the most coronagraphs. Starshades could have an IWA as small as $1 \lambda / D$. However, probing more distant systems would (1) reduce the absolute flux from the planet and (2) potentially make the angular separation fall below the IWA.

Future space-based exoplanet missions will probably be limited to $H$ band and shorter wavelengths. Beyond $H$ band, the thermal background rises, requiring a cryogenic telescope and instruments, which significantly increases the cost of the mission. For our simulations of space telescopes, we consider a wavelength range covering $0.5-1.7 \mu \mathrm{m}$. Such a wide passband poses a challenge for wavefront control, which typically operates at a bandwidth of $10 \%-20 \%$ (Trauger \& Traub 2007). In order to reach the full $0.5-1.7 \mu \mathrm{m}$ wavelength coverage, multiple observations or simultaneous wavefront control/coronagraph channels are necessary. While these practical concerns are neglected in our simulations, we note that lines for molecular species such as $\mathrm{O}_{2}$ and $\mathrm{CO}_{2}$ concentrate in smaller bands. Characterizing these molecular species may only require tailoring the instrument channels to regions of the spectrum where these specific lines are abundant.

\subsection{Simulation Setup}

We use the same Earth albedo spectrum to generate the Earth-like planet's reflection spectrum as described in Section 5.1. The BT-Settl spectrum with $T_{\text {eff }}=5800 \mathrm{~K}$ and $\log (g)=4.5$ is used as the input solar-type star spectrum. The metallicity $[\mathrm{Fe} / \mathrm{H}]$ is set to zero. The telescope and instrument parameters used are listed in Table 7 , and the planet and host star information can be found in Table 8 .

\subsection{Masked Cross-correlation}

Space-based observations offer an opportunity to detect molecular absorption bands at low spectral resolution for a large wavelength range without confusion by the Earth's atmosphere. The opportunity also comes with challenges for 
Table 7

Telescope and Instrument Parameters for LUVOIR or HabEx

\begin{tabular}{lll}
\hline \hline Parameter & \multicolumn{1}{c}{ Value } & Unit \\
\hline Telescope aperture & 4.0 or 12.0 & $\mathrm{~m}$ \\
Telescope+instrument throughput & $10 \%$ & $\ldots$ \\
Wavefront correction error floor & 5 & $\mathrm{~nm}$ \\
Spectral resolution & varied & $\ldots$ \\
Spectral range & $0.5-1.7$ & $\mu \mathrm{m}$ \\
Exposure time & 400 or 100 & $\mathrm{hour}$ \\
Fiber angular diameter & 1.0 & $\lambda / D$ \\
Readout noise & 0.0 or $2.0^{\mathrm{a}}$ & $e^{-\mathrm{a}}$ \\
Dark current & 0.0 or 0.002 or $5.5 \times 10^{-6 \mathrm{~b}}$ & $e^{-} s^{-1}$ \\
\hline
\end{tabular}

Notes.

a Based on H2RG detector specification (Blank et al. 2012) and e2v CCD specification.

${ }^{\mathrm{b}}$ Used for $\mathrm{O}_{2}$ detection.

Table 8

A Sun-Earth System at 5 pc

\begin{tabular}{lcc}
\hline \hline Parameter & Value & Unit \\
\hline Star & & \\
Effective temperature $\left(T_{\text {eff }}\right)$ & 5800 & $\mathrm{~K}$ \\
Mass & 1.0 & $M_{\odot}$ \\
Radius & 1.0 & $R_{\odot}$ \\
Surface gravity $(\log g)$ & 4.5 & $\mathrm{cgs}$ \\
Metallicity $([M / H])$ & 0.0 & $\mathrm{dex}$ \\
Distance & 5.0 & $\mathrm{pc}$ \\
Rotational velocity & 2.0 & $\mathrm{~km} \mathrm{~s}^{-1}$ \\
Inclination $(i)$ & 50 & $\mathrm{degree}$ \\
Radial velocity & 0,0 & $\mathrm{~km} \mathrm{~s}^{-1}$ \\
Planet & & \\
$V$ sin ${ }^{* * *}$ & 0.5 & $\mathrm{~km} \mathrm{~s}^{-1}$ \\
Inclination $(i)$ & 50 & $\mathrm{degree}$ \\
Semimajor axis $(a)$ & 1.0 & $\mathrm{au}$ \\
Radius & 1.0 & $R_{\oplus}$ \\
Radial velocity & 20.4 & $\mathrm{~km} \mathrm{~s}^{-1}$ \\
Illuminated area & 0.5 & $\ldots$ \\
Planet/star contrast & $6.1 \times 10^{-11}$ & $\ldots$ \\
Angular separation & 200.0 & $\mathrm{mas}$ \\
Angular separation at $1 \mu \mathrm{m}$ for $12 \mathrm{~m}$ aperture & 11.6 & $\lambda / D$ \\
Angular separation at $1 \mu \mathrm{m}$ for $4 \mathrm{~m}$ aperture & 3.9 & $\lambda / D$ \\
\hline
\end{tabular}

the cross-correlation technique. First, $\mathrm{O}_{2}$ and $\mathrm{CO}_{2}$ have only a few narrow absorption bands over a wide wavelength range. Using the entire wavelength range would not increase the CCF $\mathrm{S} / \mathrm{N}$, but instead introduce noise in the CCF. For example, cross-correlating the observed spectrum with an $\mathrm{O}_{2}$ template at low spectral resolution results in a higher peak at the $\mathrm{H}_{2} \mathrm{O}$ absorption band than at $\mathrm{O}_{2}$ bands simply because the $\mathrm{H}_{2} \mathrm{O}$ band is deeper.

For ground-based observations, all CCF definitions are observables. The CCF peak is the highest value of CCF and the CCF noise is the rms of certain parts of the CCF. While we prefer such definitions, we have to make a few adjustments in the CCF S/N calculations for the space-based case. First, we apply a masked cross-correlation method to only select wavelength regions with absorption lines (Queloz 1995; Baranne et al. 1996). This approach alleviates the confusion of the regular cross -correlation at low spectral resolutions. At a given spectral resolution, the cross-correlation mask selects wavelength regions with absorption lines or bands deeper than $1 \%$ and sets the rest of the reduced spectrum to the median value. Here, we consider a wider range of spectral resolutions from $R=25$ to $R=102,400$.

In principle, the CCF can be calculated in smaller wavelength blocks and the CCFs of different blocks can then be added with weights based on information content and $\mathrm{S} / \mathrm{N}$. However, this is impractical at low spectral resolution where there are fewer than $100 \mathrm{CCF}$ data points across the wavelength range from 0.5 to $1.7 \mu \mathrm{m}$. With fewer than 10 data points (i.e., 10 spectral block divisions), it is impossible to obtain a meaningful statistical peak and noise level. At higher spectral resolution, calculating the $\mathrm{CCF}$ over a broad range of wavelengths is essentially the same as calculating CCFs over smaller wavelength blocks followed by coadding the CCFs. In order to maintain a consistent treatment across all considered spectral resolutions, we calculate the CCF over the entire wavelength range from 0.5 to $1.7 \mu \mathrm{m}$.

\subsection{A New Definition of the CCF $S / N$}

When using the masked cross-correlation technique over a broad wavelength range, only parts of the CCF carries signal, whereas the rest is flat. As a result, the previous definition of a structure-limited CCF $\mathrm{S} / \mathrm{N}$ does not apply. In addition, the previous definition of a photon-noise-limited CCF $\mathrm{S} / \mathrm{N}$ does not apply either. The reason is that the photon noise can be different by a factor of a few times from one end of the spectrum to the other. Although the average of two ends of a spectrum could be used to calculate the photon-noise-limited CCF S/N, we choose the following way of defining the CCF $\mathrm{S} / \mathrm{N}$ for the broadband wavelength coverage case.

We simulate 100 observations, record all the CCF peak values, and make a histogram of the $\mathrm{CCF}$ peak distribution divided into 10 bins from zero to the maximum CCF peak (as shown in Figure 13). The CCF $\mathrm{S} / \mathrm{N}$ is defined as the ratio between the median of the simulated CCF peak distribution and the standard deviation of the CCF peak distribution because the histogram is a reasonable approximation to a Gaussian distribution. As a result, a significant CCF peak should have a distribution that is well separated from zero (panel (A) in Figure 13), i.e., the lowest-valued bin that includes zero should have no data point from 100 simulations. This is roughly equivalent to a $3 \sigma$ limit because there are 100 simulated peaks and none of them is consistent with zero $(p<1 \%)$. For a peak that is not well separated from zero (panel (B) in Figure 13), we mark the corresponding $\mathrm{CCF} S / \mathrm{N}$ as zero. This happens when the height of the lowest-valued bin is not zero.

If the $\mathrm{CCF}$ peak is caused by random variations rather than the planet signal (panel (C) in Figure 13), the CCF peak may be significant and well separated from zero. However, the center of the distribution of CCF peaks should be separated from the noiseless $\mathrm{CCF}$ peak. Therefore we mark the $\mathrm{CCF} \mathrm{S} / \mathrm{N}$ as zero if the noiseless CCF peak is in the first $15 \%$ or the last $15 \%$ percentile of the simulated CCF peak distribution. We choose this percentile for the following reasons. First, the boundaries are roughly consistent with the $1 \sigma$ range of the simulated $\mathrm{CCF}$ peaks (red dashed lines versus blue error bars). This ensures that the inferred CCF peak is consistent with the noiseless $\mathrm{CCF}$ peak within $1 \sigma$. Second, the first $15 \%$ percentile also roughly marks the $1 \sigma$ lower boundary of the simulated CCF peak distribution. This helps to exclude significant CCF peaks that are due to elevated noise levels with $1 \sigma$ significance (panel (C)). A higher-valued percentile cut would shrink the spacing 

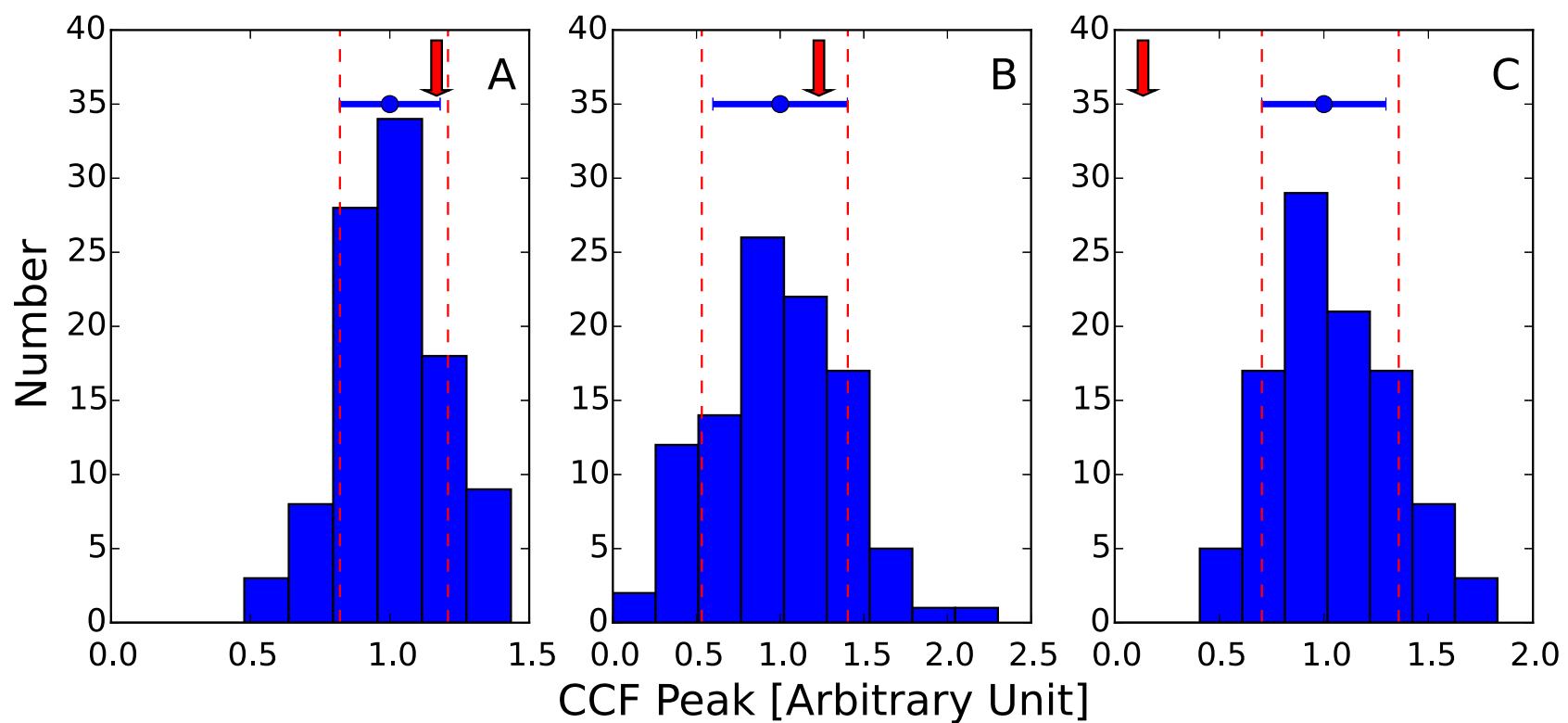

Figure 13. Distribution of simulated CCF peaks. Red arrows are values of CCF peaks in the noiseless case. Blue dots and error bars are median values and standard deviations of the distributions. Red dashed lines mark the first and last 15\% percentile of the distribution. Panel (A): the distribution of CCF peaks is well separated from zero and the median is consistent with a noiseless CCF peak value. Panel (B): the distribution of CCF peaks is not well separated from zero because the lowestvalued bin has a non-zero number. Panel (C): the distribution of CCF peaks is well separated from zero, but the median is not consistent with a noiseless CCF peak value. The CCF peaks in this case are caused by random fluctuation that is induced by noise.

between two dashed lines and therefore exclude more true positives (e.g., panel (A)). On the other hand, a lower-valued percentile cut would include more false positives caused by random noise (e.g., panel (C)).

The CCF $\mathrm{S} / \mathrm{N}$ definition used in this section requires multiple iterations to obtain a distribution of CCF peaks and to infer the significance of the CCF peak. In practice, only one CCF is obtained for one observation. In the presence of random systematics such as speckle chromatic noise at low spectral resolution, it is difficult to assess whether an observed CCF peak is caused by a planet signal or is due to random systematics. Simulations that incorporate our best knowledge of noise sources and systematics may be the only solution to quantify the detection significance.

At low spectral resolutions, the cross-correlation may not be the best possible way to detect planets or molecules. A more straightforward way is to conduct a conventional ADI/SDI sequence, detecting the planet, obtaining a low-resolution spectrum, and inferring the molecular presence by measuring the absorption band depth. However, in order to compare the instrument performance over a broad range of spectral resolutions, we are compelled to apply the same crosscorrelation technique at all spectral resolutions for consistency and comparison purposes.

\subsection{Speckle Noise and Its Chromaticity}

The spectral signature of speckles at deep star light suppression may resemble broad absorption bands that mimic features in the planet's spectrum (Krist et al. 2008). These artifacts are caused by wavelength-dependent wavefront errors after amplitude and phase correction using a wavefront control scheme, such as electric field conjugation (EFC) (Trauger \& Traub 2007; Groff et al. 2016).

To determine the HRS signature of speckles, we simulated a notional space telescope with realistic optical surface errors and a coronagraph instrument with two deformable mirrors, each

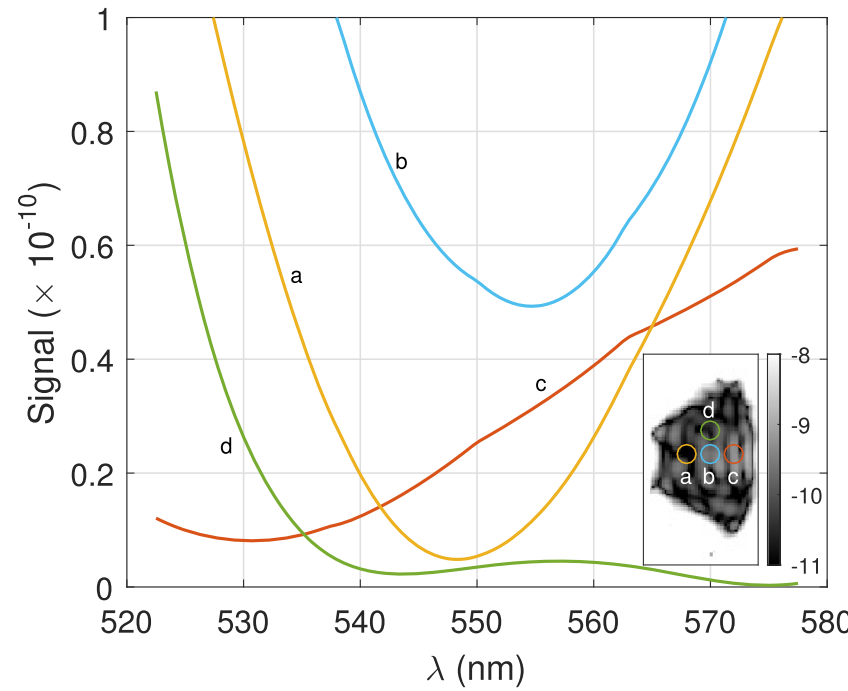

Figure 14. High-resolution spectral signature of stellar speckles in a singlemode fiber-fed spectrograph. (inset) Simulated irradiance in the dark hole, on a $\log$ scale, at $\lambda_{0}=550 \mathrm{~nm}$. The circles indicate the locations of the representative samples. All values are normalized to the peak of the stellar PSF prior to the coronagraph.

with 16 actuators across the beam diameter. A dark hole was generated in the residual star light (10\% passband about $\lambda_{0}=550 \mathrm{~nm}$ ) within a $60^{\circ}$ wedge-shaped region extending from 3-10 $\lambda / D$ using EFC (see Figure 14, inset). The fiber coupling efficiency of the stellar field was calculated assuming a single-mode fiber with a fundamental mode diameter of $\lambda / D$. Figure 14 shows the estimated stellar signal detected by the spectrograph at example locations within the dark hole, indicated by the color circles in the inset. We find that the spectra of speckles generally take the form of low-order polynomials. Since wavefront control simulations tend to be computationally intensive, we approximate this effect by generating a low-order spline function with points anchored 
at the edge of wavelength range for wavefront control and at an optimized wavelength.

The wavelength coverage of our HDC observation simulations is $0.5-1.7 \mu \mathrm{m}$. However, it is not possible to use the deformable mirrors to generate a dark hole over such a wide range, so that it is instead assumed that the wavefront control is performed at $0.1 \mu \mathrm{m}$ intervals. For each reduced planet spectrum, we inject randomly generated speckle chromatic noise to study its impact on the cross-correlation technique. To remove the low-frequency speckle chromatic noise, we apply a high-pass filter before cross-correlating with template spectrum. Some of the intrinsic planet absorption may also be removed since the absorption bands and the speckles have similar frequency content in the spectral domain.

\subsection{Simulating Large Ultraviolet, Optical, and Infrared (LUVOIR) Observations}

The LUVOIR telescope is a concept study for a large nextgeneration space telescope (Crooke et al. 2016). The size of LUVOIR is not defined yet, but will likely be in the $10-16 \mathrm{~m}$ range. Here, we conservatively select $12 \mathrm{~m}$ for our simulations. The study of exoplanets will be one of its main scientific objectives.

Unlike the ground-based simulations for HR 8799 e and 51 Eri b, we consider the photon-noise-limited case. Detector noise (both RN and dark current) is set to zero. The availability of zero-noise detectors for space-based coronagraphic missions has been a concern and has recently been identified as a technology gap. It is now subject to growing awareness and research (Rauscher et al. 2016). The impact of detector noise and speckle chromatic noise will also be discussed later in this section.

We note that the CCF structure-limited case and the mismatched-spectrum case are not considered for space-based observation. First, the CCF structure-limited case is very probably not the case for Earth-like planet observations, which are usually in the low $\mathrm{S} / \mathrm{N}$ regime. Second, there is no mismatched-spectrum case since we use the same albedo spectrum for the input planet spectrum and the template spectrum for the cross-correlation. The results shown below should therefore be interpreted as an optimistic prediction of the performance for upcoming space-based instruments and missions.

\subsubsection{Planet and Molecular Detection}

Figure 15 shows the CCF S/N contours in the phase space of star light suppression versus spectral resolution. An Earth-like planet with a planet/star contrast of $6.1 \times 10^{-11}$ can be detected at all spectral resolutions for star light suppression levels better than $2 \times 10^{-9}$ with a CCF S/N of 5 . In the spacebased photon-noise-limited regime, the detectability gain of HRS is not as significant as for the ground-based case of an Earth-like planet orbiting an $\mathrm{M}$ star. The highest spectral resolution considered $(R=102,400)$ increases the CCF S/N by a factor of $\sim 2$ when compared to lower spectral resolutions (e.g., $R=25$ ). This can be explained by analyzing Figure 16 . Even at spectral resolution as low as $R=25$, broad $\mathrm{H}_{2} \mathrm{O}$ absorption bands are resolved, and this enables detection of planet or molecular species with the cross-correlation technique. Increasing spectral resolution helps to resolve lines and thus adds an additional fine peak on the band-resolved CCF.
This additional line-resolved peak is about twice the height as the band-resolved CCF peak, which explained the factor of $\sim 2$ gain in $\mathrm{CCF} S / \mathrm{N}$.

So far, we have shown that, not surprisingly, the crosscorrelation technique works on broad molecular bands at low spectral resolution. It is interesting to note that this result comes from the fact that space observations are free of any contamination from the Earth's atmosphere. Indeed, in space, there is no need to distinguish between the signal for molecular species that the exoplanet and the Earth atmospheres might have in common. In our ground-based simulations, we apply a high-pass filter to remove the Earth's atmosphere absorption and stellar continuum low-frequency variations, which essentially removes the absorption bands from the planet signal. Therefore, ground-based simulations rely entirely on resolving absorption lines to detect the exoplanet molecular species.

However, there are two main caveats to the photon-noiselimited case. First of all, the photon-noise limit cannot realistically be reached at low spectral resolution; speckle noise and its chromaticity need to be accounted for (see Section 6.5.3). Second, not every molecular species is readily detectable at $R=25$. At a star light suppression level of $10^{-10}$, $\mathrm{O}_{2}$ and $\mathrm{CO}_{2}$ become detectable at $R=50$ with a CCF $\mathrm{S} / \mathrm{N}$ of 7.1 and 3.9, respectively. The reason is that the absorption bands for $\mathrm{O}_{2}$ and $\mathrm{CO}_{2}$ are narrower than those for $\mathrm{H}_{2} \mathrm{O}$ (see Figure 8). When the spectral resolutions are higher than $R=50$, these bands start to be resolved and the band-resolved CCF peak appears (see Figure 16).

\subsubsection{CCF at Low S/N (per Pixel) Regime}

One advantage of HDC observations is the relaxation of the requirement for the star light suppression level. However, in the hypothetical (unrealistic) photon-noise-limited regime, the relaxation of star light suppression requirements for space-based observations is less obvious than in the groundbased cases. For example, the simulation for a $K_{S^{-}}$band observation of M-dwarf planets (Figure 11) shows that the relaxation of star light suppression is 2-3 orders of magnitude. However, the relaxation is only a factor of $\sim 5$ for the space-based case (Figure 15) when tracing the contour of the CCF S/N of 5 around $10^{-9}$ star light suppression. This is consistent with our finding in Section 5.2.2 that in the photon-noise regime, the relaxation of the star light suppression level depends on the number of photons from the planet that enter the instrument.

Space-based observations of an Earth-Sun system is extreme compared to ground-based observations of Earth-M dwarf cases. Figure 17 shows a comparison of noise sources at different spectral resolutions. At the highest considered spectral resolution, there are only $2-3$ photons per pixel. The average $\mathrm{S} / \mathrm{N}$ per pixel is $\sim 1 / 30$ if only photon noise from the star and the planet is considered. At this low level of $\mathrm{S} / \mathrm{N}$ per pixel, each absorption line is very noisy. Considering the proportionality of $\mathrm{CCF} \mathrm{S/N}$ to the square root of the number of lines (Snellen et al. 2015), we only expect a modest contribution from the line-resolved CCF, as shown in Figure 16, even if the spectral resolution is high enough to resolve individual absorption lines. 

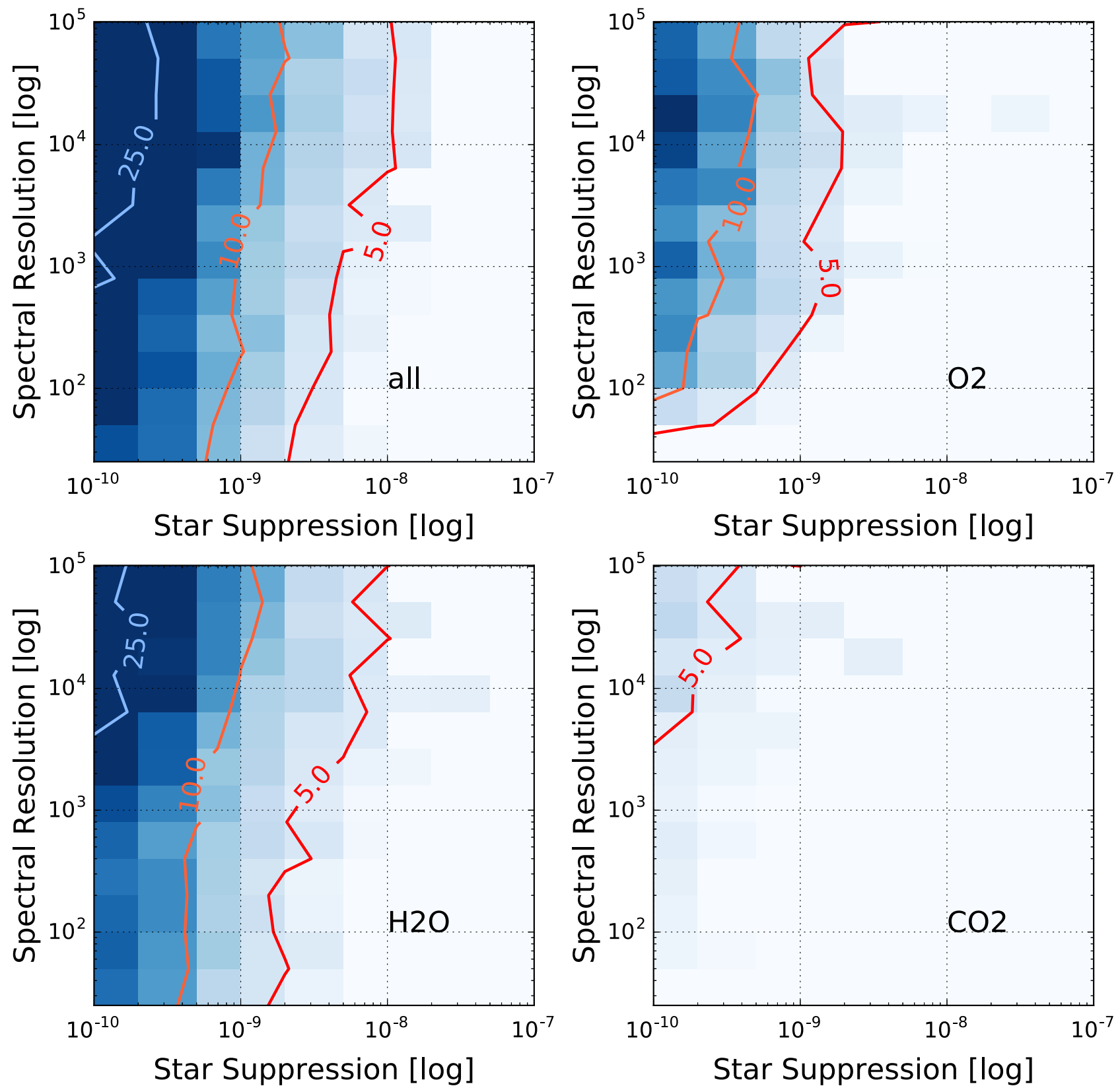

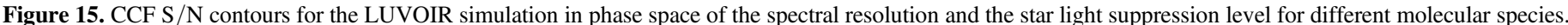
The detector noise (readout noise and dark current) is assumed to be zero. No speckle chromatic noise is considered.

\subsubsection{The Impact of Detector Noise, Speckle Noise, and Its Chromaticity}

Figure 18 shows the impact of detector noise on planet detection (see Table 7). At low spectral resolutions, the CCF $\mathrm{S} / \mathrm{N}$ contours are affected only negligibly. Contours at high spectral resolutions are significantly altered as a consequence of detector noise. Figure 17 shows that the noise contribution from dark current and $\mathrm{RN}$ for 100 readouts are comparable. When the detector noise is taken into account, the CCF $S / N$ peaks at spectral resolutions lower than $R=1000$. This implies that future space missions should not consider extreme high resolution unless detector noise can be significantly reduced, which is an active area of research (Rauscher et al. 2016). Depending on the desired CCF $\mathrm{S} / \mathrm{N}$, the requirement for star light suppression is relaxed by $1-2$ orders of magnitude compared to the astrophysical planet/star contrast, which is still very significant. The impact of detector noise on $\mathrm{O}_{2}$ and $\mathrm{H}_{2} \mathrm{O}$ detection is similar to the planet detection case, as shown in Figure 18. However, $\mathrm{CO}_{2}$ is no longer detectable after considering detector noise.

Figure 19 shows the CCF S/N contours including the effect of both detector noise and speckle chromatic noise. With a CCF $\mathrm{S} / \mathrm{N}$ greater than 5 , the performance of an HDC instrument is limited by detector noise at high spectral resolution and speckle chromatic noise at low spectral resolution. We find an optimal point at $R=1600$ where the star light suppression requirement is relaxed to $5 \times 10^{-9}$, or almost 2 orders of magnitude (the planet/star contrast is $6.1 \times 10^{-11}$ ).

\subsection{Simulating HabEX Observation}

HabEx is a concept for an exoplanet direct-imaging mission with a more modest aperture than LUVOIR (4-6.5 m). Despite 


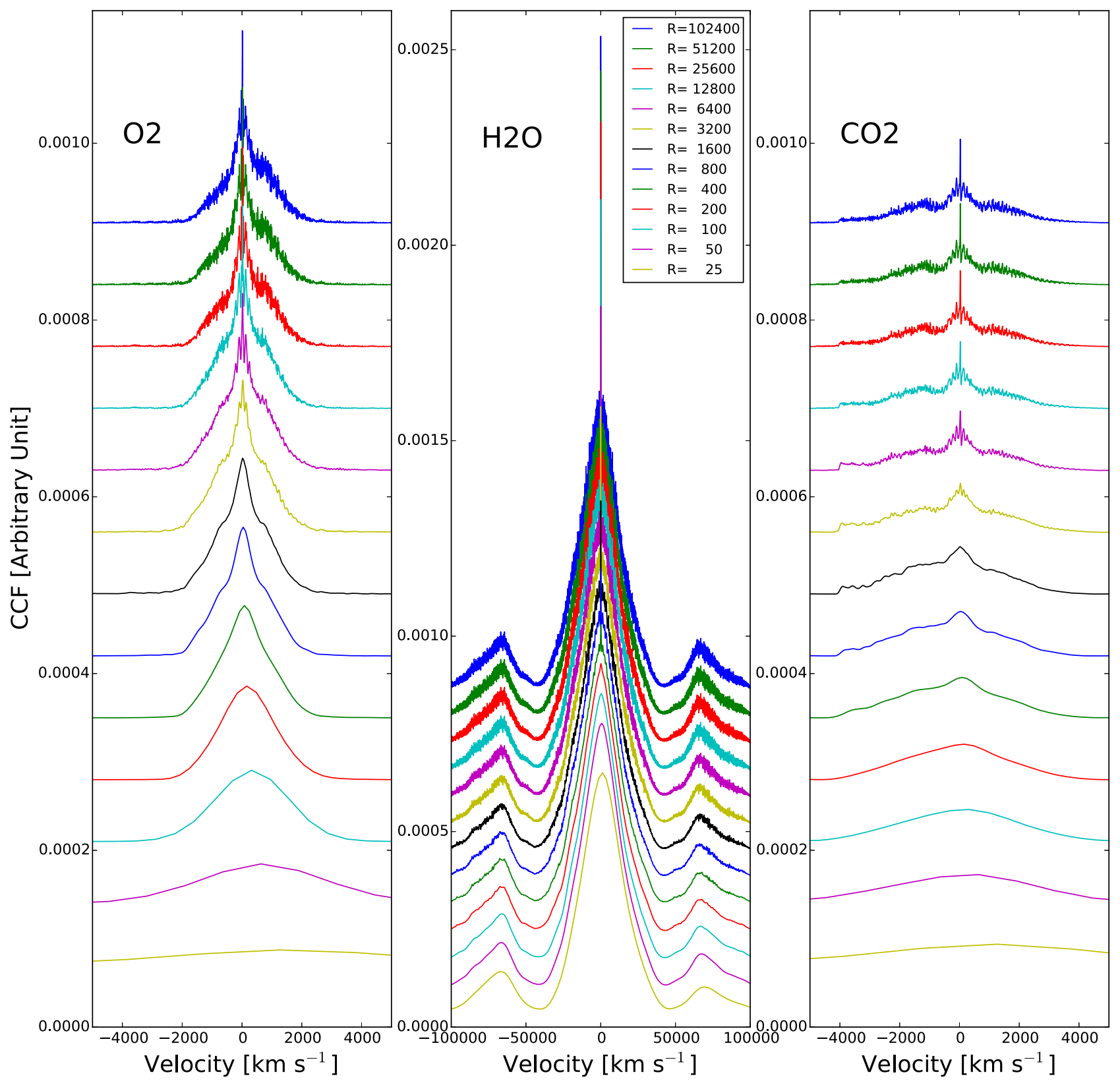

Figure 16. Cross-correlation functions for $\mathrm{O}_{2}, \mathrm{H}_{2} \mathrm{O}$, and $\mathrm{CO}_{2}$ at different spectral resolutions.

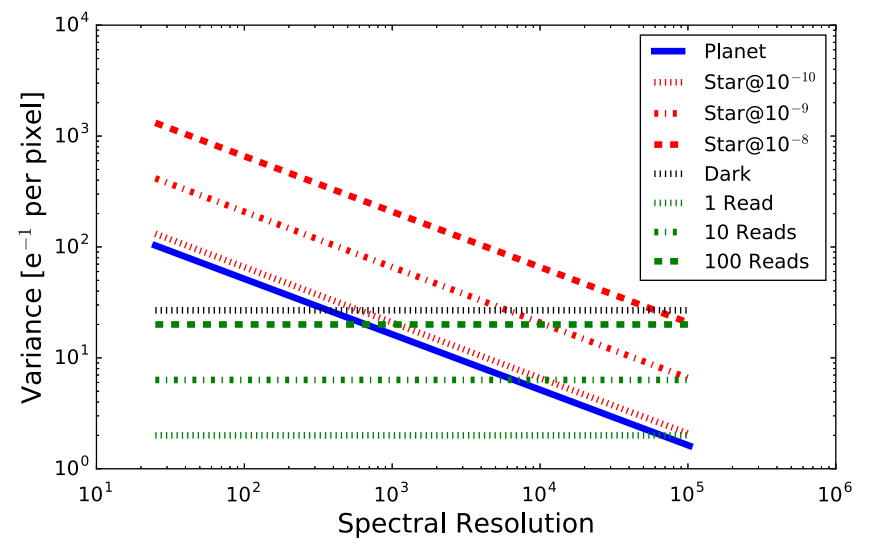

Figure 17. Comparison of noise sources at different spectral resolutions for the case of a $12 \mathrm{~m}$ space-based telescope on a Sun-Earth system at $5 \mathrm{pc}$. a smaller aperture size, HabEx has several advantages compared to LUVOIR. First, HabEx is an exoplanet-focus mission with a much larger fraction of observing time dedicated to exoplanet search and characterization. A HabEx observation can therefore afford a much longer exposure time for a single target that has a compelling case for exoplanet study. We therefore use $400 \mathrm{hr}$ total exposure time in the simulation, four times longer than what is used for the LUVOIR simulation. Second, HabEx will be optimized for exoplanet direct imaging and can potentially achieve deeper star light suppression than LUVOIR. These differences between HabEx and LUVOIR need to be considered when comparing the performance of the HDC concepts for these two missions.

Considering a conservative $4 \mathrm{~m}$ telescope diameter, we simulate HabEx observations of a Sun-Earth system at $5 \mathrm{pc}$ 


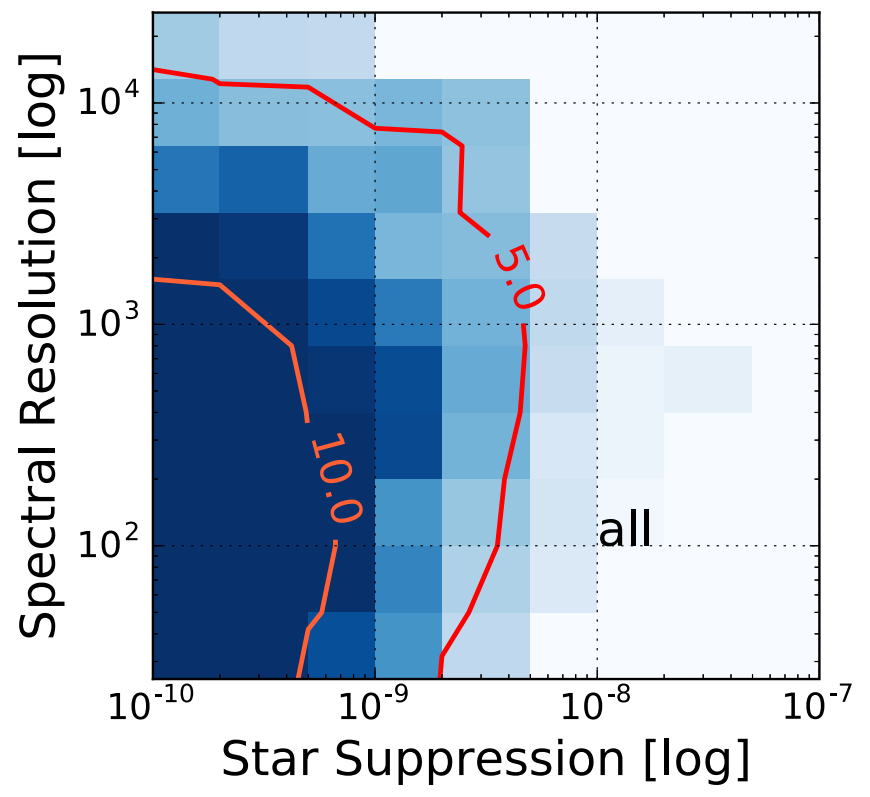

Figure 18. CCF $\mathrm{S} / \mathrm{N}$ contours for LUVOIR simulation in phase space of spectral resolution and star light suppression level for different molecular species. Detector noise (readout noise and dark current) is considered, and the values are shown in Table 7 . We assume 100 readouts during a $100 \mathrm{hr}$ observation.

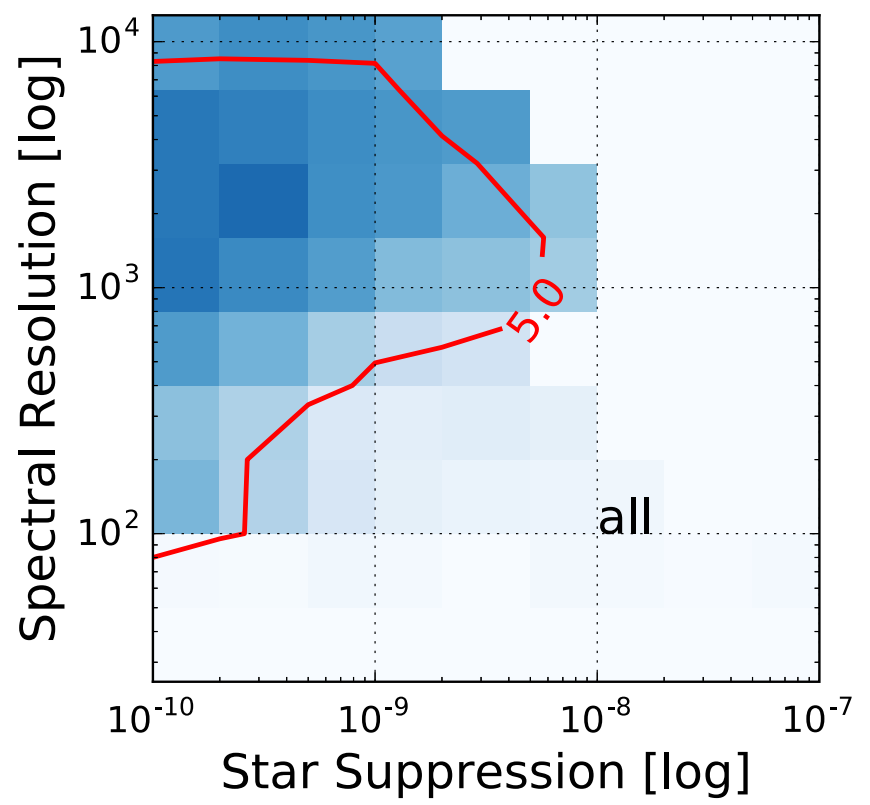

Figure 19. $\mathrm{CCF} S / \mathrm{N}$ contours for LUVOIR simulation in phase space of spectral resolution and star light suppression level for planet detection. Detector noise (readout noise and dark current) and speckle chromatic noise are considered. We assume 100 readouts during a $100 \mathrm{hr}$ observation.

with a total exposure time of $400 \mathrm{hr}$. Figure 20 shows the $\mathrm{CCF}$ $\mathrm{S} / \mathrm{N}$ contours versus spectral resolutions and star light suppression levels. The results are qualitatively similar to the LUVOIR simulation, but with a reduced CCF S/N. The reason is that the planet signal is $\sim 2$ times lower for the HabEx simulation than for the LUVOIR simulation. Although we assume an exposure time that is four times longer for HabEx observations, LUVOIR has an aperture size that is three times larger.

At $C=10^{-10}, \mathrm{H}_{2} \mathrm{O}, \mathrm{O}_{2}$, and $\mathrm{CO}_{2}$ start to be detected at $R=25, R=50$, and $R=200$ with a CCF S/N of 9.7, 5.3, and
3.1 respectively. The highest spectral resolution we consider is $R=51,200$ because there is on average less than one photon per pixel for higher spectral resolutions. If detector noise and speckle chromatic noise are considered, the optimal combination of spectral resolution and star light suppression for planet detection is $R=400$ and $C=5 \times 10^{-10}$, where the CCF S/N is 4.6 (see Figure 21). At this combination, the relaxation of the star light suppression requirement is almost a factor of $\sim 10$ (the planet/star contrast is $6.1 \times 10^{-11}$ ).

\section{Summary and Discussion}

In this paper, we presented a framework to simulate the endto-end performance of an HDC instrument. The pipeline intakes spectra of planets and stars and considers atmospheric transmission and background emission if applicable. With a realistic assumption of coronagraphic and spectroscopic system performance, the pipeline simulates observed and reduced planet spectra with reasonable noise sources including photon noise from the planet, the star, the Earth's atmosphere background emission, detector noise, and speckle chromatic noise. The pipeline also simulates the subsequent spectral analysis, such as detecting a planet and the molecular species in its atmosphere using the cross-correlation method. The pipeline can be used for a trade study of future ground-based and space-based missions dedicated to the search and characterization of exoplanets. We provide a few representative test cases: (1) observations of currently known directly imaged planets (i.e., HR 8799 e and 51 Eri b) with the $10 \mathrm{~m}$ Keck telescope; (2) observations of Proxima Cen $\mathrm{b}$ and an Earth-like planet around an M dwarf at $5 \mathrm{pc}$ with a $30 \mathrm{~m}$ class ground-based telescope; (3) observations of an EarthSun system at $5 \mathrm{pc}$ with 4 and $12 \mathrm{~m}$ space-based telescopes. These simulations are valuable in terms of understanding the power and limitation of the HDC technique.

\subsection{Lessons Learned from Simulations for Currently Known Directly Imaged Exoplanets}

We applied the pipeline to the currently known directly imaged planets HR 8799 e and 51 Eri b. We studied the feasibility of detecting such planets and characterizing the composition of their atmospheres using KPIC, a Keck HDC instrument under development. We summarize our findings as follows.

1. The $\mathrm{CCF} S / \mathrm{N}$ is not always photon noise limited. Other factors that limit the $\mathrm{CCF} S / \mathrm{N}$ include the intrinsic structure of the $\mathrm{CCF}$ and the mismatch between the observed spectrum and the template that is used in the cross-correlation (Figures 4 and 6).

2. The mismatched-spectrum case yields the lowest CCF S/N. This result highlights the importance of planet spectrum modeling in the cross-correlation method. However, the mismatched-spectrum case also represents an opportunity for atmosphere retrieval by varying model parameters to maximize the CCF peak.

3. Multiband observation is necessary in order to fully characterize the chemical composition of a planet. We considered three molecular species $\left(\mathrm{CO}, \mathrm{H}_{2} \mathrm{O}\right.$, and $\left.\mathrm{CH}_{4}\right)$ and demonstrated that the optimal band for detecting them can be different (Figures 5 and 7).

4. The increased sensitivity makes the HDC technique suitable for planet searches. For example, Lovis et al. (2017) considered an integral field unit (IFU) formed by a bundle of seven hexagonal single-mode fibers to search 

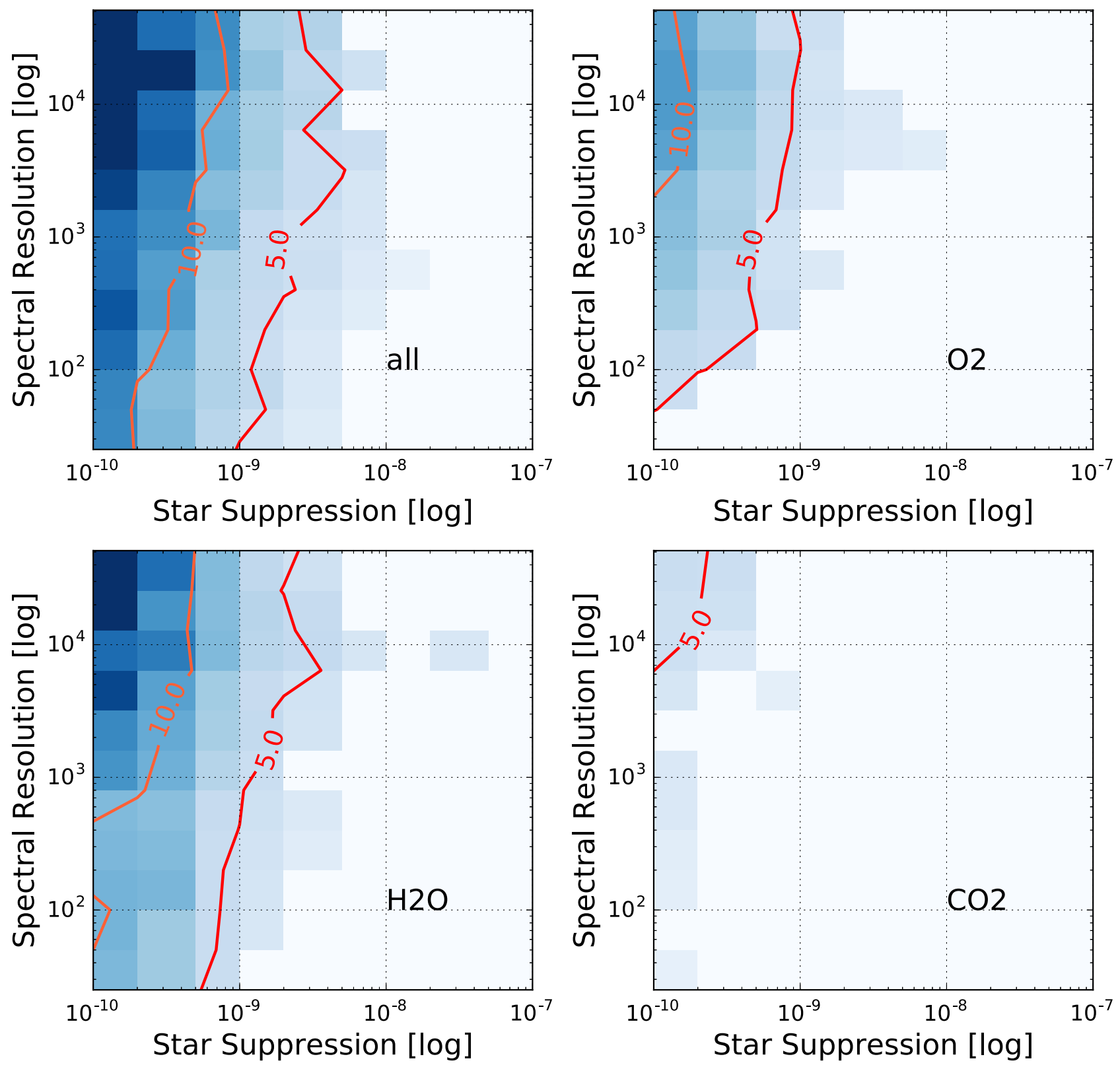

Figure 20. CCF S/N contours for the HabEx simulation in phase space of spectral resolution and star light suppression level for different molecular species. The detector noise (readout noise and dark current) is assumed to be zero. No speckle chromatic noise is considered. The total exposure time for the HabEx simulation (i.e., $400 \mathrm{hr}$ ) is four times longer than for the LUVOIR simulation. The reason is that LUVOIR is a general-purpose space mission and HabEx is an exoplanet-specific mission, which can afford a much longer exposure time on a single target.

for the exact location of Proxima Cen b. Similarly, Rains et al. (2016) proposed a 3-by-3 fiber-based IFU for planet search and characterization. METIS (Brandl et al. 2014), one of the three first-light instruments for E-ELT, will provide $L$ - and $M$-band IFU capability for HDC observations. The multiplexing capability increases the effective field of view and relaxes the requirement for pointing and tracking stability at the expense of detector size or wavelength coverage.

\subsection{Lessons Learned from Simulations for Systems with an Earth-like Planet}

HDC simulations for the observation of an Earth-like planet were pioneered by Sparks \& Ford (2002). This important topic was later on explored by a few groups (Riaud \& Schneider 2007; Kawahara \& Hirano 2014; Snellen et al. 2015; Lovis et al. 2017). The present study builds on previous work in terms of simulation method. We thoroughly explored the parameter space of spectral resolution and star light suppression level for both space-based and ground-based observations.

\subsubsection{Ground-based Observations}

Here we summarize our findings from the simulations of the ground-based observations of Earth-like planets in the habitable zone of $\mathrm{M}$ dwarfs.

1. High spectral resolution allows the star light suppression requirements for detection and characterization to be relaxed by 2-3 orders of magnitude. Using the HDC 


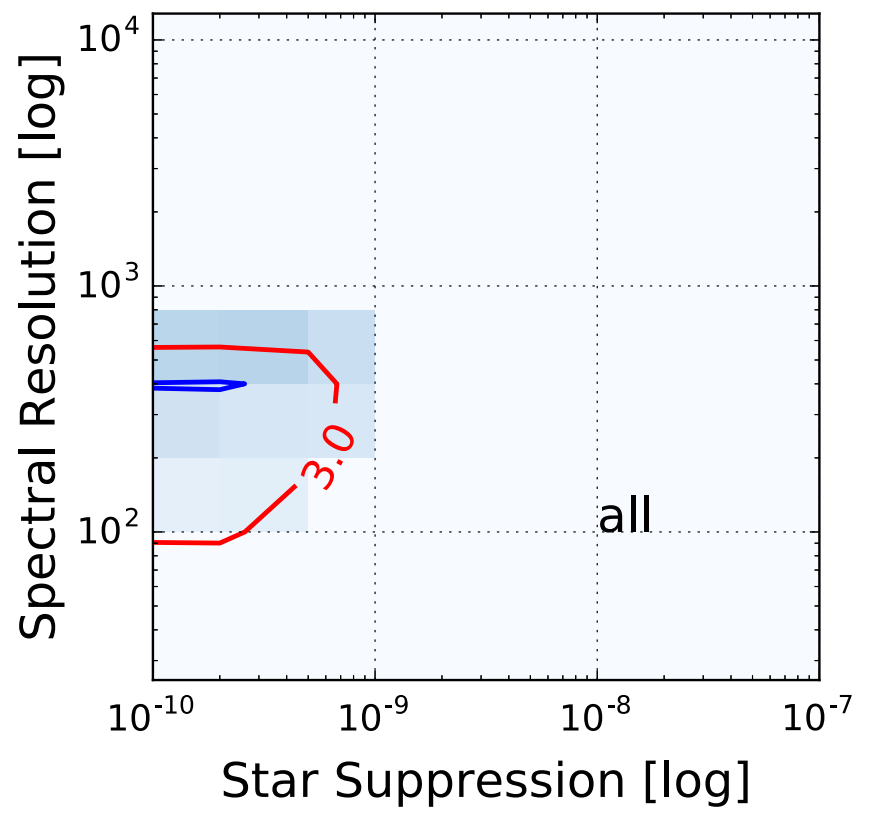

Figure 21. CCF S/N contours for HabEx simulation in phase space of spectral resolution and star light suppression level for planet detection. Detector noise (readout noise and dark current) and speckle chromatic noise are considered. We assume 400 readouts during a $400 \mathrm{hr}$ observation.

technique, we find that the minimum star light suppression level is $10^{-4}$ for the $10 \sigma$ detection of Proxima Cen $b$ with ground-based $30 \mathrm{~m}$ class telescopes.

2. In addition to Proxima Cen b, extremely large groundbased telescopes can be used to study more distant M-dwarf planet systems (e.g., 5 pc). Given the abundance of $\mathbf{M}$ dwarfs (Cantrell et al. 2013) and planets around $\mathbf{M}$ dwarfs (Dressing \& Charbonneau 2015) in the solar neighborhood within $5 \mathrm{pc}$, the prospect of studying planets around cool stars is promising.

3. The performance of an HDC instrument depends on the planet signal and its relative strength with respect to other noise sources (Figure 12). In order to reach the full potential of an HDC instrument and push for higher sensitivity at lower levels of star light suppression, increasing signal throughput from the planet is the key.

4. The dominating noise source for ground-based HDC observation with a $30 \mathrm{~m}$ class telescope is the photon noise from leaked stellar light (for $100 \mathrm{hr}$ observation, see Figure 12). Detector and sky background noise are $\sim 5$ times lower than the dominating noise source at $10^{-6}$ star light suppression level.

5. High spectral resolution $(R \sim 100,000)$ and deep star light suppression $\left(\sim 10^{-8}\right)$ offers a unique opportunity to study an exoterrestrial atmosphere at unprecedentedly high S/N (Figures 9-11), although severe technological hurdles need to be overcome.

\subsubsection{Comparison to Previous Results}

Kawahara \& Hirano (2014) predicted that $10^{-4}$ and $10^{-5}$ star light suppression levels are required for the $3 \sigma$ detection of $\mathrm{H}_{2} \mathrm{O}$. We ran a simulation with a similar setup as theirs and find that $\mathrm{H}_{2} \mathrm{O}$ can be detected with a CCF S/N of 6.8 at star light suppression level of $5 \times 10^{-4}$. We suspect that the factor of 2 difference in detection significance may be attributed to the different approach in calculating the CCF S/N.

Snellen et al. (2015) investigated the detectability of a shortperiod super-Earth around Proxima Cen and concluded that the planet can be detected with a significance of 10 for a wavelength coverage from 0.6 to $0.9 \mu \mathrm{m}$ with an HDC instrument on the E-ELT. Following the details in their paper, we found a CCF $\mathrm{S} / \mathrm{N}$ of 4.0 for such a super-Earth. We note that the star light suppression level assumed in Snellen et al. (2015) is $\sim 3.3 \times 10^{-4}$, whereas we found that a star light suppression level of $1 \times 10^{-4}$ is the minimal requirement for detection. The difference in $\mathrm{CCF} S / \mathrm{N}$ and star light suppression requirement can be explained by the spectra used in the cross-correlation. Snellen et al. (2015) considered both reflected stellar lines and planetary molecular absorption lines, whereas we considered only planetary molecular absorption lines. Within the $0.6-0.9 \mu \mathrm{m}$ wavelength coverage, the reflected stellar lines contribute more to the CCF peak than the planetary molecular absorption lines. Not considering the reflected stellar lines results in a lower $\mathrm{CCF} S / \mathrm{N}$ than reported by Snellen et al. (2015). However, in $J, H$, and $K$ band, the planetary molecular absorption lines contribute much more to the $\mathrm{CCF}$ peak than the reflected stellar lines. Considering the reflected stellar lines therefore does not significantly improve the $\mathrm{CCF} \mathrm{S} / \mathrm{N}$ in near-infrared wavelengths.

Lovis et al. (2017) investigated the potential of SPHERE + EXPRESSO on the VLT to search and characterize Prox Cen b. They found that the planet can be detected at 5- $\sigma$ with a total of $240 \mathrm{hr}$ integration time. We adopted their values in our pipeline and found that the $\mathrm{CCF} S / \mathrm{N}$ is $6.8-9.2$ for a star light suppression level of between $1 / 5000$ and $1 / 2000$.

\subsubsection{Space-based Observation}

Here we summarize our findings from the simulations of the space-based observations of Earth-like planets in the habitable zone of a solar-type star.

1. For a $12 \mathrm{~m}$ space-based telescope, an Earth-like planet can be detected at all spectral resolutions $(R=25-102$, 400) for star light suppression levels better than $2 \times 10^{-9}$ with a CCF $\mathrm{S} / \mathrm{N}$ of 5 (Figure 15). For a $4 \mathrm{~m}$ space-based telescope, the CCF $\mathrm{S} / \mathrm{N}$ reduces because of the smaller aperture size (Figure 20).

2. The number of photons from the planet entering the instrument is critical. As the aperture increases from the $4 \mathrm{~m}$ (HabEx-like telescope) to $12 \mathrm{~m}$ (LUVOIR-like telescope), we find a significant increase of CCF S/N for all molecular species for a fixed exposure time of $100 \mathrm{hr}$.

3. While the $12 \mathrm{~m}$ LUVOIR concept has an aperture size that is three times larger than that of the $4 \mathrm{~m} \mathrm{HabEx}$ concept, HabEx can afford a much longer exposure time and can potentially achieve deeper star light suppression because it is focused and optimized for exoplanet study. These differences between HabEx and LUVOIR need to be considered when comparing the performance of the HDC concepts.

4. Space-based observations can operate at low spectral resolution without the concern of contamination by the Earth's atmosphere (speckle chromaticity might be worse, however, see next point). Planet or molecular species can be detected by their absorption bands at 
spectral resolutions as low as $R=25$. In contrast, for ground-based observation, we apply a high-pass filter to remove the Earth's atmosphere absorption and stellar continuum low-frequency variations, which essentially removes the absorption bands from the planet signal. Therefore, ground-based observations rely entirely on resolving absorption lines for detection. That is the regime where HRS comes into play as a critical component.

5. The performance of an HDC instrument is limited by detector noise at high spectral resolution and speckle chromatic noise at low spectral resolution (Figures 19 and 21).

6. Future space missions should not consider extreme high resolution unless detector noise can be significantly reduced.

\subsection{Future Works}

In a future paper, we wish to establish a quantitative relationship between planet signal and relaxation of the requirements for star light suppression. In particular, we aim to answer quantitatively how the gain by HRS in an HDC instrument changes with planet signal in the presence of various noise sources. In addition, we will make the simulations more realistic by considering details in echelle spectroscopic data reduction. One outstanding question is how to preserve an extremely weak planet signal (a few to hundreds of photons per pixel) at every step of the data reduction and spectral analysis.

The authors would like to acknowledge the financial support of the Heising-Simons foundation. The authors acknowledge ideas and advice from the participants in the Exoplanet Imaging and Characterization: Coherent Differential Imaging and Signal Detection Statistics workshop organized by the W.M. Keck Institute for Space Studies.

\section{References}

Absil, O., Mawet, D., Karlsson, M., et al. 2016, Proc. SPIE, 9908, 99080Q Anglada-Escudé, G., Amado, P. J., Barnes, J., et al. 2016, Natur, 536, 437 Baines, E. K., White, R. J., Huber, D., et al. 2012, ApJ, 761, 57

Baraffe, I., Homeier, D., Allard, F., \& Chabrier, G. 2015, A\&A, 577, A42

Baranne, A., Queloz, D., Mayor, M., et al. 1996, A\&AS, 119, 373

Benneke, B. 2015, arXiv:1504.07655

Benneke, B., \& Seager, S. 2013, ApJ, 778, 153

Beuzit, J.-L., et al. 2008, Proc. SPIE, 7014, 701418

Birkby, J. L., de Kok, R. J., Brogi, M., et al. 2013, MNRAS, 436, L35

Blank, R., Anglin, S., Beletic, J. W., et al. 2012, Proc. SPIE, 8453, 845310

Bonnefoy, M., Zurlo, A., Baudino, J. L., et al. 2016, A\&A, 587, A58

Borysow, A. 2002, A\&A, 390, 779

Bottom, M., Shelton, J. C., Wallace, J. K., et al. 2016, PASP, 128, 075003

Bowler, B. P. 2016, PASP, 128, 102001

Brandl, B. R., Feldt, Ma., Glasse, A., et al. 2014, Proc. SPIE, 9147, 914721

Brogi, M., de Kok, R. J., Birkby, J. L., Schwarz, H., \& Snellen, I. A. G. 2014, A\&A, 565, A124

Brogi, M., Snellen, I. A. G., de Kok, R. J., et al. 2012, Natur, 486, 502
Brogi, M., Snellen, I. A. G., de Kok, R. J., et al. 2013, ApJ, 767, 27

Burrows, A., \& Volobuyev, M. 2003, ApJ, 583, 985

Caffau, E., Ludwig, H.-G., Bonifacio, P., et al. 2010, A\&A, 514, A92

Cantrell, J. R., Henry, T. J., \& White, R. J. 2013, AJ, 146, 99

Crooke, J. A., Roberge, A., Domagal-Goldman, S. D., et al. 2016, in Status and Path Forward for the Large Ultraviolet/Optical/Infrared Surveyor (LUVOIR) Mission Concept Study

Crossfield, I. J. M., Biller, B., Schlieder, J. E., et al. 2014, Natur, 505, 654

Currie, T., Burrows, A., Girard, J. H., et al. 2014, ApJ, 795, 133

de Kok, R. J., Brogi, M., Snellen, I. A. G., et al. 2013, A\&A, 554, A82

Des Marais, D. J., Harwit, M. O., Jucks, K. W., et al. 2002, AsBio, 2, 153

Dressing, C. D., \& Charbonneau, D. 2015, ApJ, 807, 45

Gomez Gonzalez, C. A., Absil, O., Absil, P.-A., et al. 2016, A\&A, 589, A54

Gontcharov, G. A. 2006, A\&AT, 25, 145

Groff, T. D., Eldorado Riggs, A. J., Kern, B., \& Jeremy Kasdin, N. 2016, JATIS, 2, 011009

Hu, R., Ehlmann, B. L., \& Seager, S. 2012a, ApJ, 752, 7

Hu, R., \& Seager, S. 2014, ApJ, 784, 63

Hu, R., Seager, S., \& Bains, W. 2012b, ApJ, 761, 166

Hu, R., Seager, S., \& Bains, W. 2013, ApJ, 769, 6

Huby, E., Baudoz, P., Mawet, D., \& Absil, O. 2015, A\&A, 584, A74

Kawahara, H., \& Hirano, T. 2014, arXiv: 1409.5740

Kaye, A. B., \& Strassmeier, K. G. 1998, MNRAS, 294, L35

Kempton, E. M.-R., Perna, R., \& Heng, K. 2014, ApJ, 795, 24

Konopacky, Q. M., Barman, T. S., Macintosh, B. A., \& Marois, C. 2013, Sci, 339, 1398

Krist, J. E., Shaklan, S. B., \& Levine, M. B. 2008, Proc. SPIE, 7010, 701044

Lockwood, A. C., Johnson, J. A., Bender, C. F., et al. 2014, ApJL, 783, L29

Lord, S. D. 1992, NASA Technical Memorandum, 103957

Lovis, C., Snellen, I., Mouillet, D., et al. 2017, A\&A, 599, A16

Macintosh, B., Graham, J. R., Barman, T., et al. 2015, Sci, 350, 64

Macintosh, B., Graham, J. R., Ingraham, P., et al. 2014, PNAS, 111, 12661

Maire, A.-L., Skemer, A. J., Hinz, P. M., et al. 2015, A\&A, 576, A133

Martin, E. C., Fitzgerald, M. P., McLean, I. S., et al. 2014, Proc. SPIE, 9147, 914781

Mawet, D., Wizinowich, P., Dekany, R., et al. 2016, Proc. SPIE, 9909, 99090D Millar-Blanchaer, M. A., Graham, J. R., Pueyo, L., et al. 2015, ApJ, 811, 18 Oppenheimer, B. R., Baranec, C., Beichman, C., et al. 2013, ApJ, 768, 24

Piskunov, N. E., Kupka, F., Ryabchikova, T. A., Weiss, W. W., \& Jeffery, C. S. 1995, A\&AS, 112, 525

Pueyo, L., Soummer, R., Hoffmann, J., et al. 2015, ApJ, 803, 31

Queloz, D. 1995, in IAU Symp. 167, New Developments in Array Technology and Applications, ed. A. G. D. Philip, K. Janes, \& A. R. Upgren, 221

Rains, A. D., Ireland, M. J., Jovanovic, N., et al. 2016, Proc. SPIE, 9908, 990876

Rajan, A., Barman, T., Soummer, R., et al. 2015, ApJL, 809, L33

Rauscher, B. J., Canavan, E. R., Moseley, S. H., Sadleir, J. E., \& Stevenson, T. 2016, arXiv: 1607.05708

Riaud, P., \& Schneider, J. 2007, A\&A, 469, 355

Rothman, L. S., \& Gordon, I. E. 2009, in 64th Int. Symp. Molecular Spectroscopy, Abstract, \#RI01, https://molspect.chemistry.ohio-state.edu/ symposium_64/symposium/

Schwarz, H., Ginski, C., de Kok, R. J., et al. 2016, A\&A, 593, A74

Snellen, I. A. G., Brandl, B. R., de Kok, R. J., et al. 2014, Natur, 509, 63

Snellen, I. A. G., de Kok, R. J., de Mooij, E. J. W., \& Albrecht, S. 2010, Natur, 465,1049

Snellen, I., de Kok, R., Birkby, J. L., et al. 2015, A\&A, 576, A59

Soummer, R., Brendan Hagan, J., Pueyo, L., et al. 2011, ApJ, 741, 55

Sparks, W. B., \& Ford, H. C. 2002, ApJ, 578, 543

Tennyson, J., \& Yurchenko, S. N. 2012, MNRAS, 425, 21

Trauger, J. T., \& Traub, W. A. 2007, Natur, 446, 771

van Leeuwen, F. 2007, A\&A, 474, 653

Wagner, K., Apai, D., Kasper, M., et al. 2016, Sci, 353, 673

Wright, D. J., Chené, A.-N., De Cat, P., et al. 2011, ApJL, 728, L20

Zurlo, A., Vigan, A., Galicher, R., et al. 2016, A\&A, 587, A57 